\title{
Two component dark matter with multi-Higgs portals
}

\author{
Ligong Bian, ${ }^{a}$ Tianjun Li, ${ }^{a, b}$ Jing Shu ${ }^{a}$ and Xiao-Chuan Wang ${ }^{a}$ \\ ${ }^{a}$ State Key Laboratory of Theoretical Physics and Kavli Institute for Theoretical Physics China, \\ Institute of Theoretical Physics, Chinese Academy of Sciences, \\ Beijing 100190, P. R. China \\ ${ }^{b}$ School of Physical Electronics, University of Electronic Science and Technology of China, \\ Chengdu 610054, P. R. China \\ E-mail: lgb@itp.ac.cn, tli@itp.ac.cn, jshu@itp.ac.cn, xcwang@itp.ac.cn
}

ABSTRACT: With the assistance of two extra groups, i.e., an extra hidden gauge group $\mathrm{SU}(2)_{D}$ and a global U(1) group, we propose a two component dark matter (DM) model. After the symmetry $\mathrm{SU}(2)_{D} \times \mathrm{U}(1)$ being broken, we obtain both the vector and scalar DM candidates. The two DM candidates communicate with the standard model (SM) via three Higgs as multi-Higgs portals. The three Higgs are mixing states of the SM Higgs, the Higgs of the hidden sector and real part of a supplement complex scalar singlet. We study relic density and direct detection of DM in three scenarios. The resonance behaviors and interplay between the two component DM candidates are represented through investigating of the relic density in the parameter spaces of the two DMs masses. The electroweak precision parameters constrains the two Higgs portals couplings $\left(\lambda_{m}\right.$ and $\left.\delta_{2}\right)$. The relevant vacuum stability and naturalness problem in the parameter space of $\lambda_{m}$ and $\delta_{2}$ are studied as well. The model could alleviate these two problems in some parameter spaces under the constraints of electroweak precision observables and Higgs indirect search.

KeYwords: Beyond Standard Model, Cosmology of Theories beyond the SM

ARXIV EPRINT: 1412.5443 


\section{Contents}

1 Introduction 1

2 The model 3

3 Dark matter analysis 5

3.1 Relic density analysis $\quad 6$

$\begin{array}{lll}3.2 & \text { Direct detection } & 10\end{array}$

4 Higgs indirect search and electroweak precision constraints 11

$\begin{array}{lll}4.1 & \text { Higgs indirect search } & 12\end{array}$

$\begin{array}{lll}4.2 & \text { Electroweak precision observables constraints } & 13\end{array}$

5 Vacuum stability 14

6 Footprint of the naturalness problem $\quad \mathbf{1 5}$

$\begin{array}{ll}\text { 6.1 The naturalness problem } & 16\end{array}$

$\begin{array}{ll}\text { 6.2 Indirect search for the scenario which alleviates the naturalness problem } & 17\end{array}$

$\begin{array}{lll}7 & \text { Discussions and conclusion } & 18\end{array}$

$\begin{array}{lr}\text { A Annihilation cross sections } & 19\end{array}$

$\begin{array}{ll}\text { B Boltzman equations } & 21\end{array}$

C One loop $\beta$ functions $\quad 22$

\section{Introduction}

The discovery of Higgs particle with mass around $126 \mathrm{GeV}$ at Large hadron collider (LHC) [1, 2], roughly consistent with the SM predictions, seems complete the SM. However, the naturalness problem is still unsolved. To alleviate the problem, as well known and extensively studied, more bosonic fields are needed [3-7]. Recently, it has been noticed that the Higgs field-strength renormalization could be enhanced by these new boson fields [8-10]. And that after the Higgs field normalization, the Higgs coupling's modification could be detected indirectly $[9,11]$. Thus, it shed light on testing the mechanisms which alleviates the naturalness problem. Secondly, the SM has to be extended in order to accommodate the cold dark matter (CDM) and baryon number asymmetry of the universe (BAU). In order that the BAU not being washed out after its generation, the strong first order electroweak phase transition (SFOEWPT) is necessary, which is out of the capacity 
of the SM and new scalar fields are needed [12-14]. ${ }^{1}$ At the same time, the way to test the mechanism alleviating the naturalness problem could also been used to test SFOEWPT, which is just one of the preliminary aims of the International Linear Collider (ILC) [8]. Thirdly, vacuum instability could be rescued by introducing vector and scalar fields [3, 1722 ], and thus making the inflation with not very small top quark mass possible to coincide with the BICEP and Plank data [23, 24].

All above arguments require us to extend the SM with new bosonic freedoms, which we choose vector and scalar fields. There are many studies on the multi-component DM scenarios, see [3, 25-35], as well as the dynamical DM scenario [36, 37] whose phenomenological consequences are often quite distinct and can be applied to a much broader variety of multi-component DM scenarios [38-41]. The primary purpose of this work is to explain the CDM relic density, where the two different DM components interact with each other besides with the three Higgses, thus affects the evolution of the DM components number densities through the coupled Boltzman equations [3, 42].

For the method to introduce vector dark matter through the effective Lagarangian method yielding strongly constraints on the parameters space from the unitarity constraints $[3,43,44]$, we consider the scenario in which the vector dark matter fields $\left(V^{\mu}\right)$ respects an extra non-Abelian gauge symmetry $\mathrm{SU}(2)_{D}$. After the $\mathrm{SU}(2)_{D}$ being broken via the the complex $\operatorname{doublet}(\phi)$, one $\mathrm{SO}(3)$ symmetry is induced which $V^{\mu}$ respects to, thus making $V^{\mu}$ stable. $^{2}$ One more complex scalar singlet, $\mathbb{S}$, is supplemented to the model. After the global U(1) symmetry, i.e., $\mathbb{S} \rightarrow e^{i \alpha} \mathbb{S}$ respected by $V(H, \phi, \mathbb{S})$, being broken spontaneously and softly, the real part of $\mathbb{S}(S)$ gets a vacuum expectation value(VEV), and the imaginary part of $\mathbb{S}(A)$ respects the reduced $Z_{2}$ symmetry, which makes the other DM candidate. After the breaking of the $\mathrm{SU}(2)_{D} \times \mathrm{U}(1)$, the Higgs field of the SM mixed with two scalar fields, the real parts of $\phi\left(\eta^{\prime}\right)$ and $S$. Since the two component DMs interact with the SM particles and each other through the three Higgs fields, one could expect three resonance enhancement effects. And three significant enlargement of the magnitude of the annihilation cross sections, of the DM to DM and the DM to SM particles, appears. The magnitudes of the relic density around the three resonances decrease, since which is robustly inversely proportional to the annihilation cross sections of the DM to SM particles. The magnitudes of annihilation cross sections of scalar DM fields (vector DM fields) to the SM particles, around the three Higgs field masses, are enlarged due to the $t, u$ and seagull channels of $A A(V V) \rightarrow h_{i} h_{i} \cdot{ }^{3}$ The spin-independent (SI) DM-nucleon scattering cross sections are determined by $t$-channel interactions between the nucleon and two component DMs through the exchangeing of three Higgs fields. The model could be distinguished from the model with no interaction between the two DMs, since the opening of the channel $A A \rightarrow V V(V V \rightarrow A A)$ does affect the evolution of DM number density, and thus the magnitude of relic density. This kind of effects are explored and illustrated in DM relic density analysis section of this work. The Higgs indirect search at the LHC requires small

\footnotetext{
${ }^{1}$ In fact, additional heavy fermions could also make the SFOEWPT feasible, see $[15,16]$.

${ }^{2}$ The hidden gauge theory has also been used to study self-interacting dark matter [46, 47], inflation [48], and the model with a kinetic mixing portal between the gauge boson DM and SM [45].

${ }^{3}$ Hereafter, the Lorenz index $\mu$ of the vector field $V^{\mu}$ will be hided for simplicity in some cases.
} 
mixing between $h$ and $\eta^{\prime}(S)$. The electroweak precision observable experiment imposes stringent constraints on our parameter spaces. The vacuum stability of the model could be improved under the above two considerations. We explored the way to alleviate the naturalness problem and its' indirect search as well.

The paper is organized as follows. We construct the model and explore relic density and direct detection of the DMs. After which, we investigate the Higgs indirect search and electroweak precision constraints on the model. And then the stability of the model are studied. We also consider the possibility to alleviate naturalness problem and the way to trace the footprint of which. At last, we conclude this work with discussions and conclusion.

\section{The model}

To construct the model which includes the stable vector and scalar fields, we introduce these two fields as follows. The vector field $V^{\prime \mu}$ is introduced as the gauged field of the $\mathrm{SU}(2)_{D}$ symmetry, which couples to the SM through a doublet, $\phi$, which is a singlet of the $\mathrm{SM}$ but charged under the $\mathrm{SU}(2)_{D}$. Here, one should note that the mixing between $V^{\prime \mu}$ and the SM gauge bosons through kinetic mixing is absent for the non-abelian character of the $\mathrm{SU}(2)_{D}$. We supplement one more complex singlet $\mathbb{S}$. And one more global $\mathrm{U}(1)$ symmetry is required besides the $\mathrm{SU}(2)_{D}$ and the SM group. The complex singlet, which interacts with the $\mathrm{SM}$ and the $\mathrm{SU}(2)_{D}$ through the Higgs portal and $\phi$ portal terms in the scalar potential, transforms trivially under the $\mathrm{SM}$ and $\mathrm{SU}(2)_{D}$ gauge group.

The Lagrangian of our model is

$$
\begin{aligned}
& \mathcal{L}=\mathcal{L}^{S M}-\frac{1}{4} F^{\prime \mu \nu} \cdot F_{\mu \nu}^{\prime}+\left(D_{\mu} \phi\right)^{\dagger}\left(D^{\mu} \phi\right)-\frac{\mu_{\phi}^{2}}{2} \phi^{\dagger} \phi-\frac{\lambda_{\phi}}{4}\left(\phi^{\dagger} \phi\right)^{2} \\
& +\frac{b_{2}}{2}|\mathbb{S}|^{2}+\frac{d_{2}}{4}|\mathbb{S}|^{4}+\left(\frac{1}{4} b_{1} e^{i \phi_{b 1}} \mathbb{S}^{2}+a_{1} e^{i \phi_{a 1} \mathbb{S}}+\text { c.c. }\right) \\
& +V_{H, \phi, \mathbb{S}}
\end{aligned}
$$

with

$$
V_{H, \phi, \mathbb{S}}=V_{H, \phi}+V_{H, S}+V_{\phi, S}
$$

and

$$
\begin{aligned}
V_{H, \phi} & =\lambda_{m} \phi^{\dagger} \phi H^{\dagger} H, \\
V_{H, S} & =\frac{\delta_{2}}{2} H^{\dagger} H|\mathbb{S}|^{2}, \\
V_{\phi, S} & =\frac{\delta_{1}}{2} \phi^{\dagger} \phi|\mathbb{S}|^{2},
\end{aligned}
$$

where $D^{\mu} \phi=\partial^{\mu} \phi-i \frac{g_{\phi}}{2} \tau \cdot V^{\mu}$. The hidden gauge coupling $g_{\phi}<4 \pi$, required by the unitarity bound, need to be hold for any thermal particle whose relic density arises from the freeze-out of its annihilation $[43,49,50]$. In particular, $b_{1}$ and $a_{1}$ terms break the global U(1) symmetry explicitly. In the SM Lagrangian, the Higgs potential notations are 
defined as: $\mathcal{L}^{S M} \ni-\left(m^{2} / 2\right) H^{\dagger} H-\lambda\left(H^{\dagger} H\right)^{2}$ with $H=(0, v+h) / \sqrt{2}$, where $v$ is the VEV of the Higgs field.

After the $\mathrm{SU}(2)_{D}$ being spontaneously broken, both $\phi$ and the singlet $\mathbb{S}$ get the VEVs:

$$
\begin{aligned}
\phi & =\left(0, \frac{v_{\phi}+\eta^{\prime}}{\sqrt{2}}\right), \\
\mathbb{S} & =\frac{1}{\sqrt{2}}\left(v_{s}+S+i A\right) .
\end{aligned}
$$

So the eq. (2.1) in the unitary gauge recast as,

$$
\begin{aligned}
\mathcal{L}= & \mathcal{L}_{S M}-\frac{1}{4} F_{\mu \nu} \cdot F^{\mu \nu}+\frac{1}{8}\left(g_{\phi} v_{\phi}\right)^{2} V_{\mu} \cdot V^{\mu} \\
& +\frac{1}{8} g_{\phi}^{2} V_{\mu} \cdot V^{\mu} \eta^{\prime 2}+\frac{1}{4} g_{\phi}^{2} v_{\phi} V_{\mu} \cdot V^{\mu} \eta^{\prime}+\frac{1}{2}\left(\partial_{\mu} \eta^{\prime}\right)^{2} \\
& +V_{h, \eta^{\prime}, S, A},
\end{aligned}
$$

here $V^{\mu}=U V^{\prime \mu} U^{-1}-\frac{i}{g}\left[\partial_{\mu} U\right] U^{-1}$ with $U=\exp \left(-i \tau \cdot \xi / v_{\phi}\right)$. The vector DM mass is given by $m_{V}=g_{\phi} v_{\phi} / 2$, and the tree-level potential $V_{0}\left(h, \eta^{\prime}, S, A\right)$ recast as,

$$
\begin{aligned}
V_{0}\left(h, \eta^{\prime}, S, A\right)= & \frac{m^{2}}{4}(h+v)^{2}+\frac{\mu_{\phi}^{2}}{4}\left(\eta^{\prime}+v_{\phi}\right)^{2}-\frac{\lambda_{\phi}}{16}\left(\eta^{\prime}+v_{\phi}\right)^{4}+\frac{1}{4} \lambda(h+v)^{4} \\
& +\frac{1}{4} \lambda_{m}(h+v)^{2}\left(\eta^{\prime}+v_{\phi}\right)^{2}+\frac{1}{8}\left(\delta_{2}(h+v)^{2}+\delta_{1}\left(\eta^{\prime}+v_{\phi}\right)^{2}\right)\left(\left(S+v_{s}\right)^{2}+A^{2}\right) \\
& +\frac{1}{4}\left(b_{2}-b_{1}\right)\left(S+v_{s}\right)^{2}+\frac{1}{4}\left(b_{2}+b_{1}\right) A^{2}-\sqrt{2} a_{1}\left(S+v_{s}\right)+\frac{d_{2}}{8}\left(S+v_{s}\right)^{2} A^{2} \\
& +\frac{d_{2}}{16}\left(\left(S+v_{s}\right)^{4}+A^{4}\right) .
\end{aligned}
$$

Here, we would like to mention that, $\eta^{\prime}$ lives in the fundamental representation of $\mathrm{SU}(2)_{D}$, and displays a custodial symmetry $\mathrm{SO}(3)$ in the $V_{1,2,3}^{\mu}$ component space, which makes three $V_{i}^{\mu}$ components degenerate in mass and thus stable [51]. The explicit $Z_{2}$-breaking term is proportional to $a_{1}$ and being introduced here to avoid the cosmological domain wall problem [52-54]. After choosing $\phi_{a_{1}}=\phi_{b_{1}}=\pi$, the potential retains a $Z_{2}$ symmetry for $\operatorname{Im}(\mathbb{S})$, thereby ensuring the stability of the particle $A[55,56]$.

Requiring that the potential in eq. (2.9) has a minimum at $\langle H\rangle=h / \sqrt{2}=0$ and $\langle\mathbb{S}\rangle=S+i A=0+i \cdot 0$, the following minimization conditions are obtained:

$$
\frac{\partial V_{0}}{\partial h}=0, \quad \frac{\partial V_{0}}{\partial \eta}=0, \quad \frac{\partial V_{0}}{\partial S}=0, \quad \frac{\partial V_{0}}{\partial A}=0,
$$

where all derivatives are evaluated at $(h, \eta, S, A)=(0,0,0,0)$. These minimization conditions allow the Higgs VEV $v$ and the singlet VEV $v_{s}$ to replace $m^{2}$ and $b_{2}$ according to

$$
\begin{aligned}
m^{2} & \equiv-\frac{1}{2}\left(4 v^{2} \lambda+v_{s}^{2} \delta_{2}-2 v_{\phi}^{2} \lambda_{m}\right), \\
\mu_{\phi}^{2} & \equiv-\frac{1}{2}\left(\delta_{1} v_{s}+2 \lambda_{m} v^{2}+\lambda_{\phi} v_{\phi}^{2}\right), \\
b_{2} & \equiv \frac{1}{2 v_{s}}\left(4 \sqrt{2} a_{1}-v_{s}\left(-2 b_{1}+d_{2} v_{s}^{2}+\delta_{1} v_{\phi}^{2}+\delta_{2} v^{2}\right)\right) .
\end{aligned}
$$



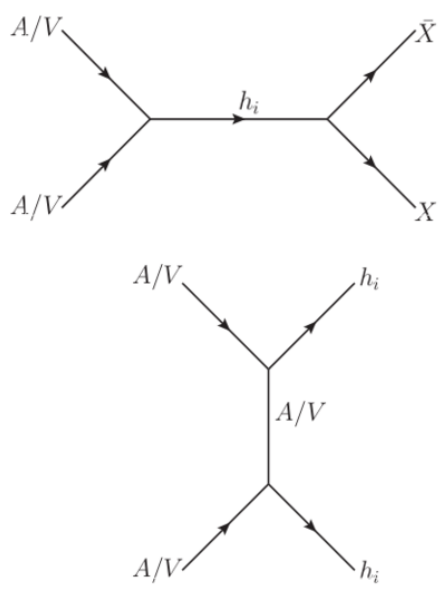
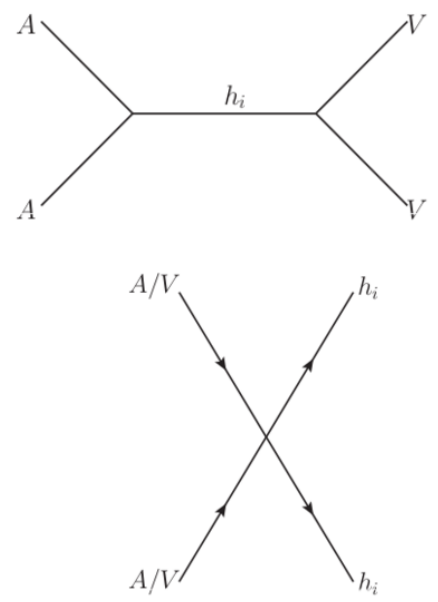

Figure 1. Feynman diagrams of annihilation channels.

Thus, at the minima, the mass matrix is obtained as

$$
M=\left(\begin{array}{cccc}
2 \lambda v^{2} & \lambda_{m} v v_{\phi} & \frac{1}{2} \delta_{2} v_{s} v & 0 \\
\lambda_{m} v v_{\phi} & \frac{1}{2} \lambda_{\phi} v_{\phi}^{2} & \frac{1}{2} \delta_{1} v_{s} v_{\phi} & 0 \\
\frac{1}{2} \delta_{2} v_{s} v & \frac{1}{2} \delta_{1} v_{s} v_{\phi} & \frac{2 \sqrt{2} a_{1}+v_{s}^{3} d_{2}}{v_{s}} & 0 \\
0 & 0 & 0 & \frac{a_{1}}{2 \sqrt{2} v_{s}}+b_{1}
\end{array}\right)
$$

And in the basis of $\left(h, \eta^{\prime}, S\right)$, we have

$$
M=\left(\begin{array}{ccc}
2 \lambda v^{2} & \lambda_{m} v v_{\phi} & \frac{1}{2} \delta_{2} v_{s} v \\
\lambda_{m} v v_{\phi} & \frac{1}{2} \lambda_{\phi} v_{\phi}^{2} & \frac{1}{2} \delta_{1} v_{s} v_{\phi} \\
\frac{1}{2} \delta_{2} v_{s} v & \frac{1}{2} \delta_{1} v_{s} v_{\phi} & \frac{2 \sqrt{2} a_{1}+v_{s}^{3} d_{2}}{v_{s}}
\end{array}\right) .
$$

To work in the mass eigenstates, i.e., $h_{1,2,3}$, we diagonalise the mass matrix eq. (2.13) through

$$
R M^{2} R^{T}=M_{\text {diag }}^{2}
$$

with matrix $R$ being given by

$$
R=\left(\begin{array}{ccc}
c_{1} c_{3} & c_{3} s_{1} & s_{3} \\
c_{2} s_{1}-c_{1} s_{2} s_{3} & c_{1} c_{2}-s_{1} s_{2} s_{3} & c_{3} s_{2} \\
s_{1} s_{2}-c_{1} c_{2} s_{3} & c_{1} s_{2}-c_{2} s_{1} s_{3} & c_{2} c_{3}
\end{array}\right),
$$

$c, s$ and their subscripts $1,2,3$ represent $\cos$, sin, $\theta_{12}, \theta_{23}$, and $\theta_{13}$ individually.

\section{Dark matter analysis}

In our model, we have two component DMs. Thus, to present the novelty of the model properly, we use the coupled boltzman equations explored in [3], with annihilation channels being depicted in figure 1, and details of annihilation cross sections are listed in section A. 


\begin{tabular}{|c|c|c|c|c|c|c|c|c|c|}
\hline$v$ & $v_{\phi}$ & $v_{s}$ & $\lambda$ & $\lambda_{\phi}$ & $d_{2}$ & $\delta_{1}$ & $\delta_{2}$ & $\lambda_{m}$ & $g_{\phi}$ \\
\hline 246 & 738 & 123 & 0.515 & 0.2 & 0.2 & 0.02 & 0.04 & 0.01 & 0.04 \\
\hline
\end{tabular}

Table 1. The input parameters for the analyses of the cases of $m_{A}>m_{V}$ and $m_{A}<m_{V}$.

\begin{tabular}{|c|c|c|c|c|c|c|}
\hline$v$ & $v_{\phi}$ & $v_{s}$ & $\lambda$ & $\lambda_{\phi}$ & $d_{2}$ & $\delta_{1}$ \\
\hline 246 & 738 & 123 & 0.515 & 0.2 & 0.21 & 0.02 \\
\hline
\end{tabular}

Table 2. The VEVs of three scalar fields and other couplings.

The relic density of each component DM could be obtained through $\Omega_{A, V} h^{2}=2.755 \times$ $10^{8} \frac{M_{A, V}}{\mathrm{GeV}} Y_{A, V}\left(T_{0}\right)[57,58]$ after we calculated $Y_{A, V}\left(T_{0}\right)$ numerically, and the total relic density is the sum of the two component DMs, $\Omega h^{2}=\Omega_{A} h^{2}+\Omega_{V} h^{2}$. As for the numerical calculations of $Y_{A, V}\left(T_{0}\right)$, we refer to eqs. (B.1), (B.2), (B.3), and (B.4).

In order to investigate how does the interplay between the two DM components affect the evolution of the DM abundance and the dependence of the DM relic density on each parameters, we scan the parameter spaces according to the following three groups:

1. Scan in the $M_{A}-M_{V}$ plane for the case of $M_{A}>M_{V}$, with the other parameters being given in table 1 .

2. Scan in the $M_{A}-M_{V}$ plane for the case of $M_{A}<M_{V}$, with the other parameters being given in table 1 .

3. Scan in the $\lambda_{m}-\delta_{2}$ plane, with DM masses being fixed as: $M_{V}=120 \mathrm{GeV}$, $M_{A}=150 \mathrm{GeV}$, and the other parameters are given in table 2 .

\subsection{Relic density analysis}

Set free parameters as in table 1, the three Higgs masses are obtained as $m_{h_{1}}=$ 124.8, $m_{h_{2}}=233.8$, and $m_{h_{3}}=71.1 \mathrm{GeV}$ in order.

\section{The first group with $m_{A}>m_{V}$}

The annihilation channel $A A \rightarrow V V$ is opened up and shown by the green line on the left panel of figure 2. The bandwidth of the green line comes from the change of the vector DM mass $m_{V}$, and the bandwidth becomes wider and wider with the increasing of scalar DM mass $m_{A}$, since the bigger the scalar DM mass $m_{A}$ the larger viable range will be left for $m_{V}$. Figure 2 depicts that the channel $A A \rightarrow X X$ is the dominate one when $m_{A}>70 \mathrm{GeV}$, and the variation tendency of the relic density $\Omega_{A} h^{2}$ is plotted in the right panel. Three peaks of the annihilation cross section of $A A \rightarrow V V$ are presented, at $m_{A}=m_{h_{1}} / 2, m_{h_{2}} / 2, m_{h_{3}} / 2$, those are the three resonances at these mass values, as could be seen from eq. (A.6) as well. At the same time, we find three other peaks of the annihilation cross section of $A A \rightarrow X X$, besides the same three peaks as that of $A A \rightarrow V V$, at $m_{A}=m_{h_{i}}$ (the three masses of three Higgs), because the new partial $t, u$ and seagull channels $A A \rightarrow h_{i} h_{i}$ through exchanging the scalar DM particle themselves are opened up around these mass values. These peaks 

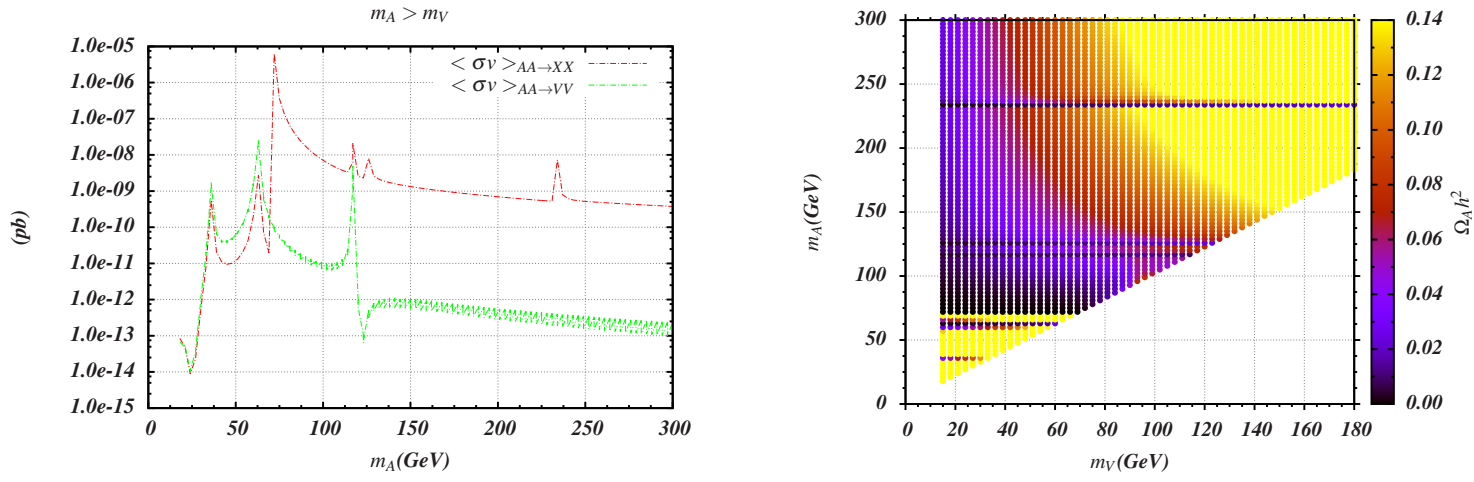

Figure 2. Left: Plots for the cross sections of channels $A A \rightarrow X X$ and $A A \rightarrow V V$ in units of $0.3894 \times 10^{9} p b$; Right: The corresponding relic density $\Omega_{A} h^{2}$.
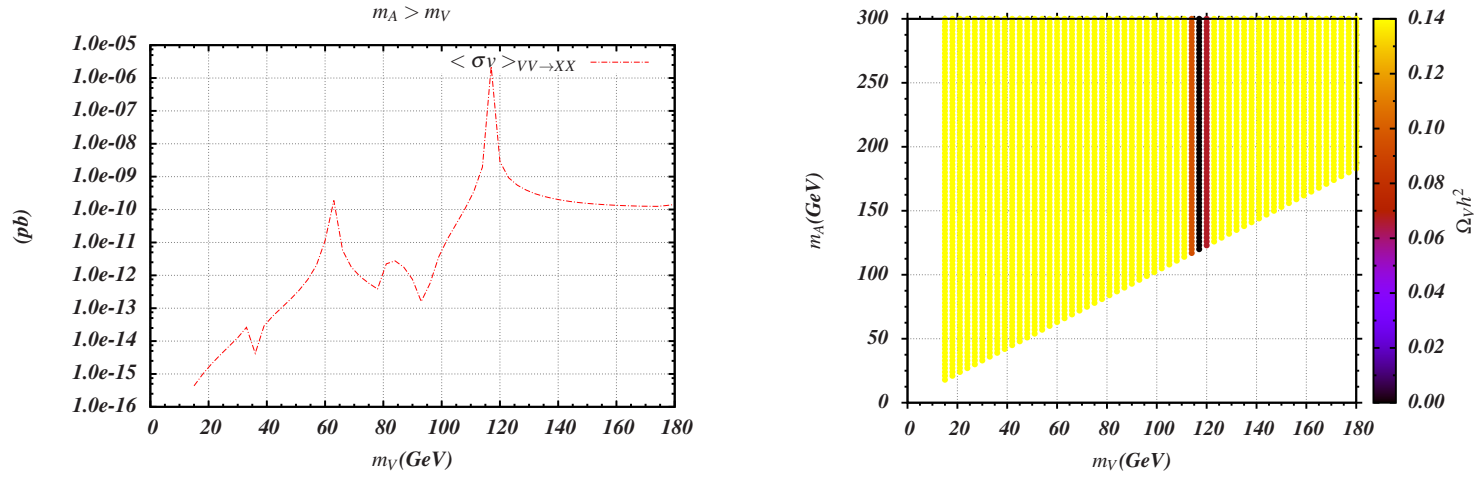

Figure 3. Left: Plot of the cross sections of channel $V V \rightarrow X X$ in units of $0.3894 \times 10^{9} \mathrm{pb}$; Right: Corresponding relic density $\Omega_{V} h^{2}$ for $m_{A}>m_{V}$.

represent themselves on the right panel of figure 2 by the transition of the color along axis of $m_{A}$. When there is a peak bigger than about $1 \times 10^{-9}$ for the cross section (on the left panel), a decrease behavior of the corresponding relic density appears on the right panel.

Last but not the least, we would like to emphase that the opening of the channel $A A \rightarrow V V$ does cause the decrease of the magnitude of $\Omega_{A} h^{2}$. Because of $\Omega_{A} h^{2} \sim$ $1 /\langle\sigma v\rangle_{A A \rightarrow X X}$, when the channel $A A \rightarrow V V$ has been shut down, one may expect $\langle\sigma v\rangle_{A A \rightarrow X X} \sim p b$ and $\Omega_{A} h^{2} \sim 0.1$. While, the right panel of figure 2 depicts more decrease of the magnitude of $\Omega_{A} h^{2}$ around $m_{A}=m_{h_{1,2}} / 2$, which demonstrates the effects of the annihilation process $A A \rightarrow V V$.

For the case of $m_{A}>m_{V}$, as shown in figure 3, the channel of $V V \rightarrow A A$ is closed when we analyzing $\Omega_{V} h^{2}$. The cross section of the channel $A A \rightarrow V V$ together with that of $V V \rightarrow X X$ affect the corresponding relic density $\Omega_{V} h^{2}$, and the second one dominates the value of $\Omega_{V} h^{2}$. Only the slim peak caused by the resonance at about $m_{V}=m_{h_{2}} / 2$ brings a sizable decreasing of $\Omega_{V} h^{2}$. The viable region of $m_{V}$ 

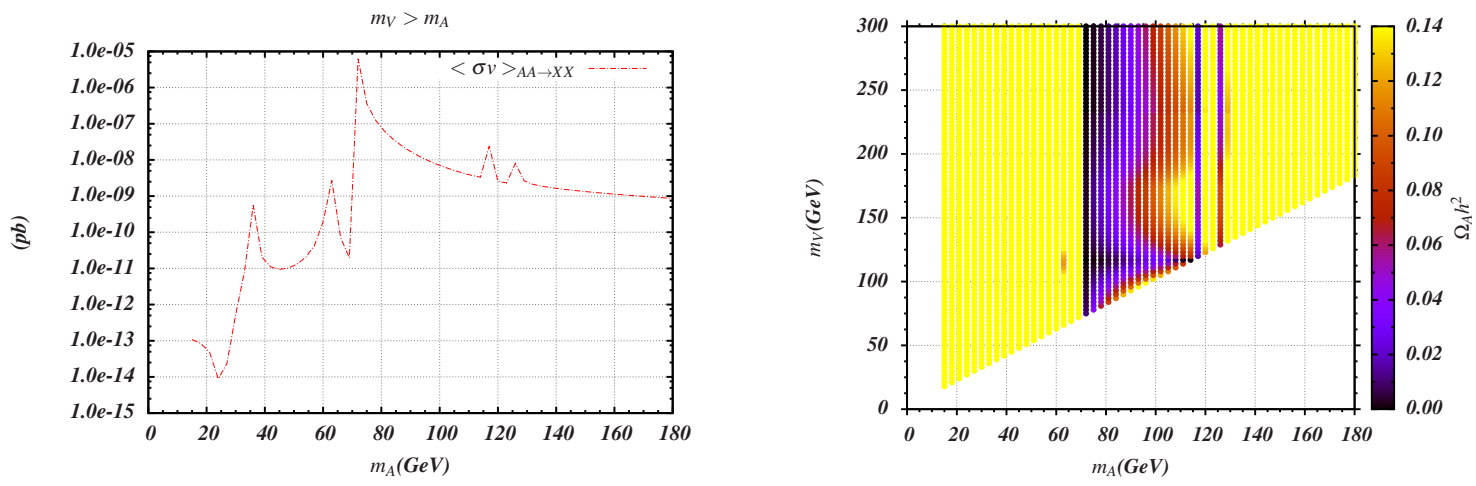

Figure 4. $m_{V}>m_{A}$. Left: Plots for annihilation cross section of channel $A A \rightarrow X X$ in units of $0.3894 \times 10^{9} p b ;$ Right: Relic density $\Omega_{A} h^{2}$.
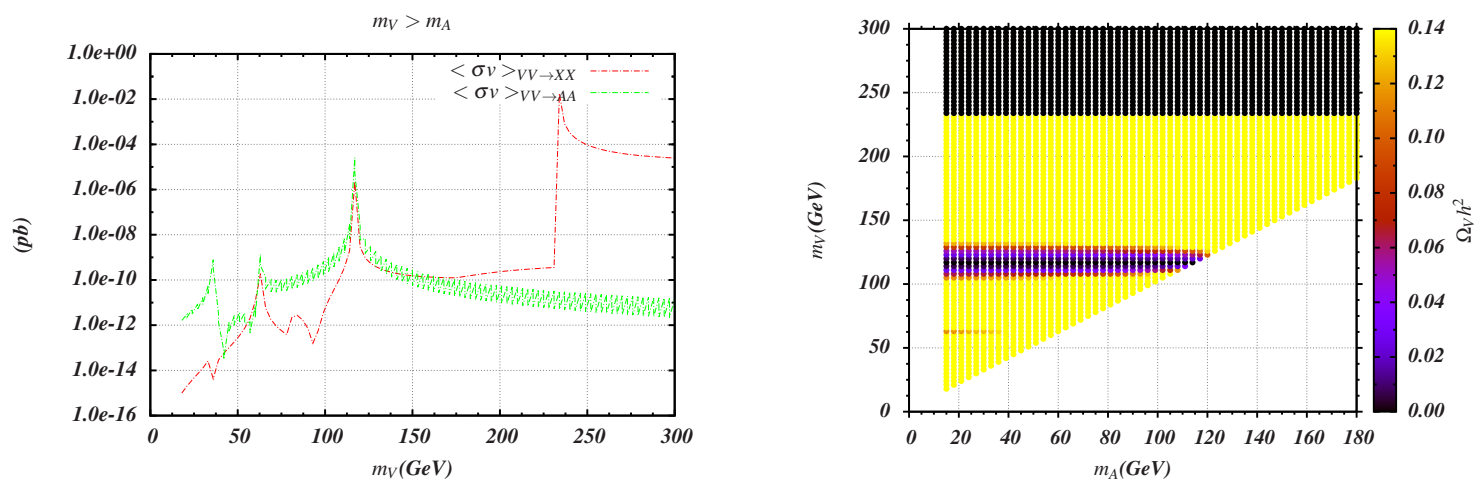

Figure 5. $m_{V}>m_{A}$. Left: Plots for the cross sections of annihilation channels $V V \rightarrow X X$ and $V V \rightarrow A A$ in units of $0.3894 \times 10^{9} p b$; Right: The dominating relic density $\Omega_{V} h^{2}$.

matching the experimental value $\Omega h^{2}=0.1189[59]\left(\Omega_{V} h^{2}\right)$ is very small as shown in the right panel.

\section{The second group with $m_{V}>m_{A}$}

In this case, the channel $A A \rightarrow V V$ is forbidden due to $m_{V}>m_{A}$, and the behavior of the cross section of the channel $A A \rightarrow X X$ is given in the left panel of figure 4 . With the increasing of $m_{A}$, the first peak caused by the resonance effect does not leave any trails, as could be seen in the magnitude of the corresponding relic density (see the right panel of figure 4), because the magnitude of the cross section is too small. The second peak is caused by the resonance effect at $m_{A}=m_{h_{1}} / 2$, and the tinny increasing of cross section induces a small decreasing of the corresponding relic density. The third peak demonstrates the opening up of the partial $t$ and $u$ channels for $A A \rightarrow h_{3} h_{3}$ and also brings a very big decrease of the relic density at about $m_{A} \geq m_{h_{3}}$ as expected. And the fourth peak, which is caused by the resonance at $m_{A}=m_{h_{2}} / 2$, brings a sizable decrease of the magnitude of the relic density also. We also notice the small waves at about $m_{V}=160 \mathrm{GeV}$ in the right panel of figure 4 . The 

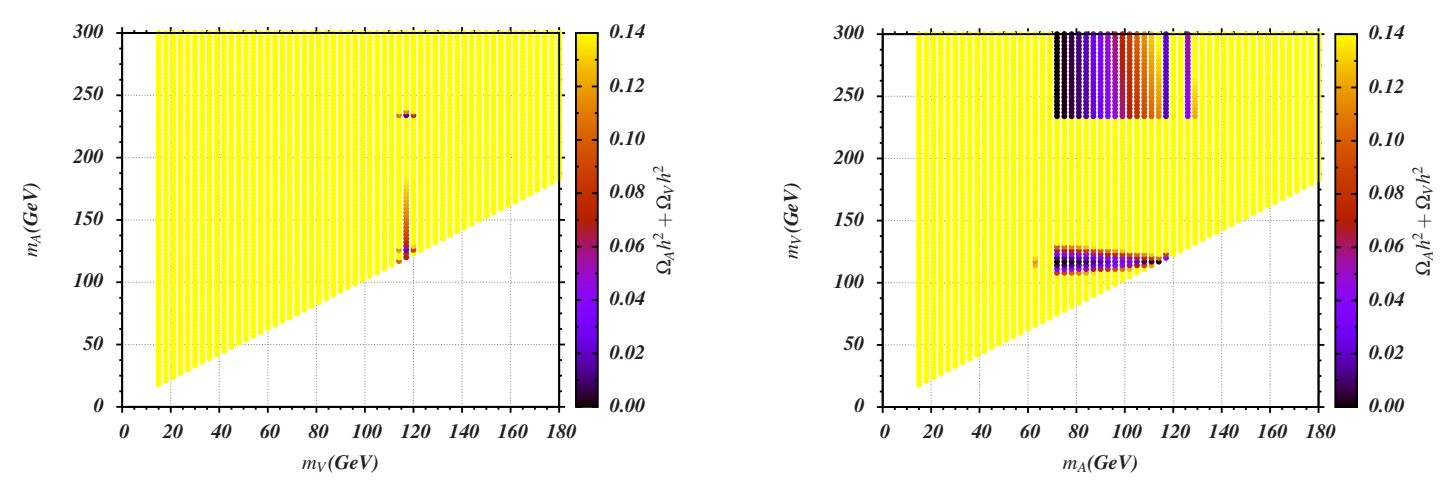

Figure 6. Plots of the total relic density $\Omega_{A} h^{2}+\Omega_{V} h^{2}$ for the case of $m_{A}>m_{V}$ and $m_{V}>m_{A}$.
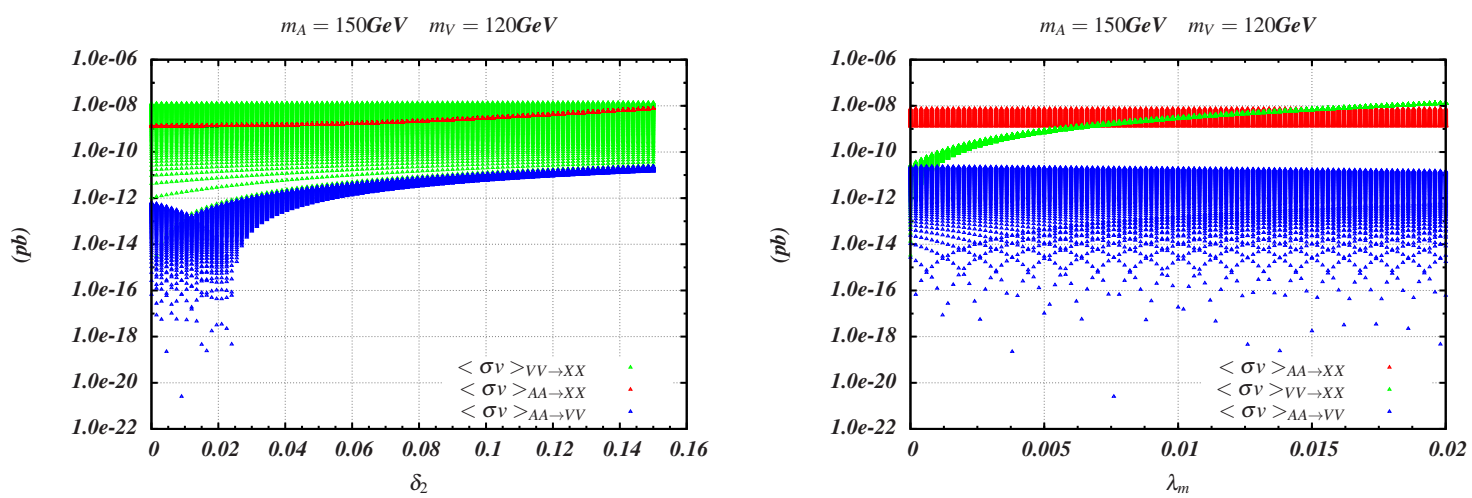

Figure 7. Plots of $\delta_{2}$ and $\lambda_{m} v s$. cross sections.

phenomenon could be explained by eq. (B.4), which illustrates that the behavior of the cross section $\langle\sigma v\rangle_{V V \rightarrow A A}$ affects the calculation of $\Omega_{A} h^{2}$ and the variation of the magnitude of the cross section $\langle\sigma v\rangle_{V V \rightarrow A A}$ generates these small waves. The channel $V V \rightarrow A A$ also causes a bigger decrease of the magnitude of $\Omega_{V} h^{2}$ in comparison with the scenario in which the channel $V V \rightarrow A A$ has been shut down, as shown in the right panel of figure 5 , especially around $m_{V} \sim m_{h_{1}} / 2\left(m_{h_{1}}\right)$.

Figure 5 is plotted to show the magnitude of $\Omega_{V} h^{2}$ contributing from both channels $V V \rightarrow X X$ and $V V \rightarrow A A$. The cross sections are small for most regions, and a big decrease of $\Omega_{V} h^{2}$ exists in the two regions $m_{V} \sim m_{h_{2}} / 2$ and $m_{V}>m_{h_{2}}$. For the first decrease, both channels induce comparable effects. And for the second decrease, it is the channel $V V \rightarrow X X$ that dominates the magnitude of $\Omega_{V} h^{2}$. Especially when $m_{V}>m_{h_{2}}$, the partial $t, u$ and seagull channels $V V \rightarrow h_{2} h_{2}$ through exchanging the vector DM particle are opened up. These effects give rise to too big $\langle\sigma v\rangle_{V V \rightarrow X X}$, and then the door to generate relic density $\Omega_{V} h^{2}$ is almost closed.

At last, the two panels in the figure 6 presents the total relic density for $m_{A}>m_{V}$ and $m_{V}>m_{A}$, respectively. Under the set of parameters as in the table 1 , the large enough magnitude of the relic density could be obtained easily. 

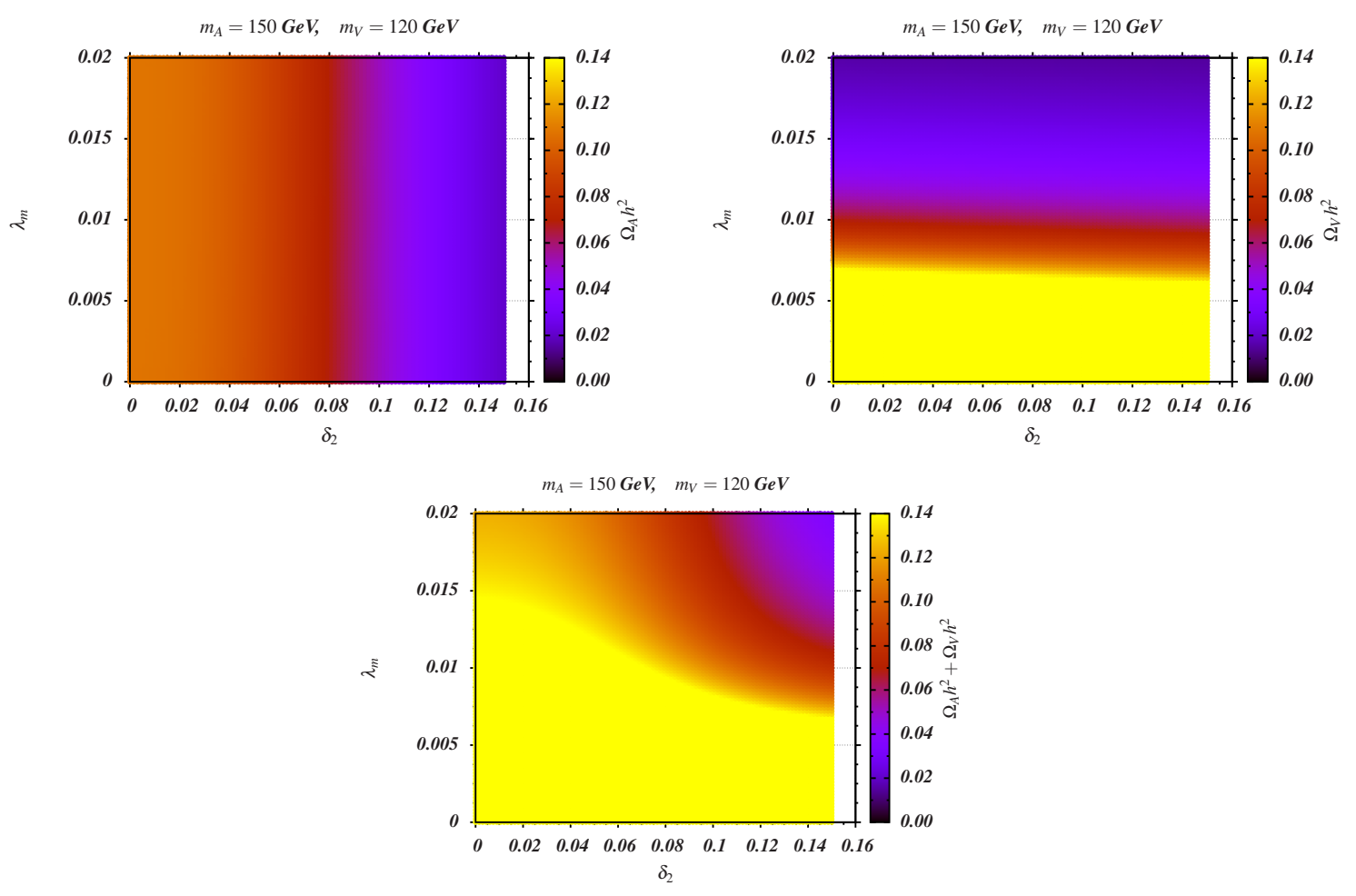

Figure 8. Plots of $\delta_{2}$ and $\lambda_{m} v s$. relic density.

\section{The third group: varying $\lambda_{m}$ and $\delta_{2}$}

Figure 7 depicts the relationships among $\delta_{2}, \lambda_{m}$ and the cross sections. Figure 8 illustrates that the magnitude of $\Omega_{A} h^{2}\left(\Omega_{V} h^{2}\right)$ reaches its critical value around $\delta_{2} \sim 0.09\left(\lambda_{m} \sim 0.01\right)$. And that the DM relic density for each component almost depends on their own coupling parameters, this is caused by the fact that all related annihilation cross sections are proportional to $\delta_{2}$ or $\lambda_{m}$.

\subsection{Direct detection}

In our model, the two component DMs, i.e., the scalar $A$ and vector $V$, interact with the SM particles through the exchange of three Higgs bosons. Thus, the DM-nucleon scattering cross section is spin-independent (SI). For each single component, the SI DM-nucleon cross sections are calculated to be

$$
\begin{aligned}
& \sigma_{S I}^{A}=\frac{1}{16 \pi v^{2}} \frac{m_{N}^{4} f_{N}^{2}}{\left(m_{A}+m_{N}\right)^{2}}\left|\frac{\delta_{2}}{2} v \frac{R_{11}}{m_{h_{1}}^{2}}+\frac{\delta_{1}}{2} v_{\phi} \frac{R_{21}}{m_{h_{2}}^{2}}+\frac{d_{2}}{2} v_{s} \frac{R_{31}}{m_{h_{3}}^{2}}\right|^{2}, \\
& \sigma_{S I}^{V}=\frac{1}{16 \pi v^{2}} \frac{m_{N}^{4} f_{N}^{2}}{\left(m_{V}+m_{N}\right)^{2}}\left|g_{\phi}^{2} v_{\phi} \frac{R_{21} R_{11}}{m_{h_{1}}^{2}}+g_{\phi}^{2} v_{\phi} \frac{R_{22} R_{21}}{m_{h_{2}}^{2}}+g_{\phi}^{2} v_{\phi} \frac{R_{23} R_{31}}{m_{h_{3}}^{2}}\right|^{2},
\end{aligned}
$$

with $R_{i j}$ coming from eq. (2.15), $m_{N}$ and $f_{N}=\sum f_{L}+3 \times \frac{2}{27} f_{H}$ are the nucleon mass and effective Higgs-nucleon coupling respectively, where $f_{N}$ is the summation of light quark 

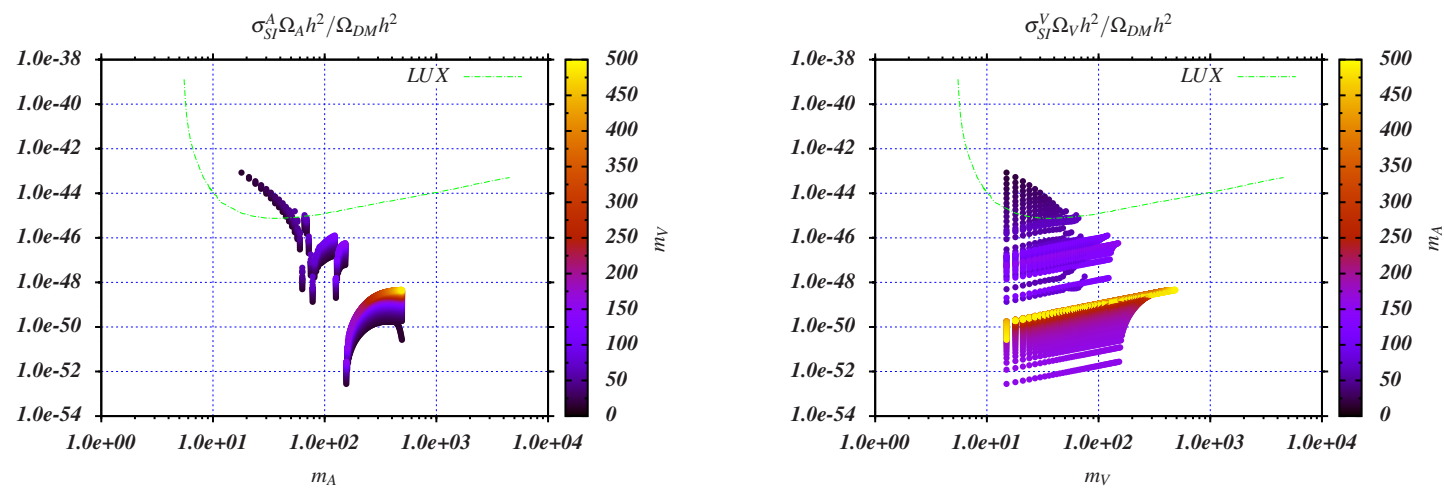

Figure 9. For the case of $m_{A}>m_{V}$, parameter regions being left considering the direct detection constraint of LUX.

$\left(f_{L}\right)$ and heavy quark $\left(f_{H}\right)$ contributions, and we take the value $f_{N}=0.326$ [60] in our numerical analysis. Since the current experiments assume that the local DM density is provided by one single DM specie, the situation that both $A, V$ components contribute to the local DM density making us unable to use the current experimental results directly. Assuming the contribution of each DM component to the local density is the same as their contribution to the relic density, the SI scattering cross section should be rescaled by a factor $\Omega_{A, V} h^{2} / \Omega_{D M} h^{2}$. Thus, the corresponding upper limit on the SI DM-nucleon cross section of each single component is $[42,56]$ :

$$
\begin{aligned}
& \sigma_{S I}^{A} \leq\left(\Omega_{D M} h^{2} / \Omega_{S} h^{2}\right) \sigma_{S I}^{\exp }\left(M_{A}\right), \\
& \sigma_{S I}^{V} \leq\left(\Omega_{D M} h^{2} / \Omega_{V} h^{2}\right) \sigma_{S I}^{\exp }\left(M_{V}\right) .
\end{aligned}
$$

Similar to the above section, here we do analyses using the LUX experiment results [61] via three groups as well.

1. $m_{A}>m_{V}$

The left and right panels of figure 9 depict that: with the value of $m_{V}$ being unbounded, $m_{A}$ should be bigger than $30 \mathrm{GeV}$.

2. $m_{V}>m_{A}$

The left and right panels of figure 10 depict that $m_{A, V}$ should be no smaller than $50 \mathrm{GeV}$.

3. parameter space of $\delta_{2}$ and $\lambda_{m}$

Figure 11 depicts that all the parameter spaces satisfy the experimental constraints.

\section{Higgs indirect search and electroweak precision constraints}

In this section, we study the effects of the mixing among the three fields $h, \eta^{\prime}, S$ using the Higgs indirect search and constraints from electroweak precision observables. 

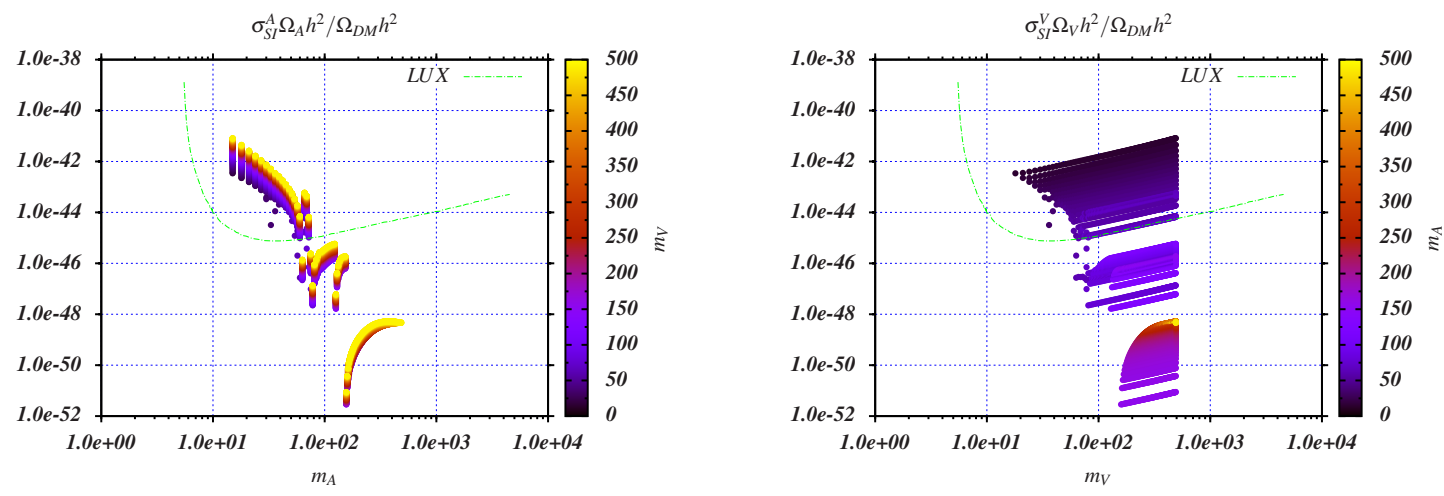

Figure 10. For the case of $m_{V}>m_{A}$, parameter regions being left considering the direct detection constraint of LUX.
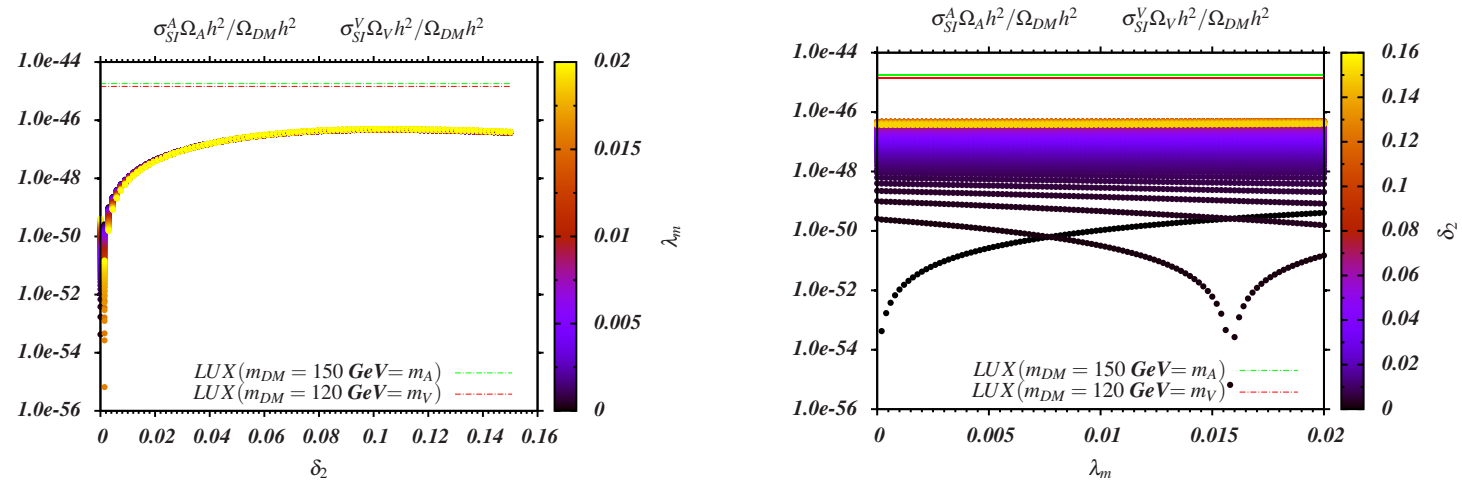

Figure 11. $\delta_{2}$ and $\lambda_{m}$. Parameter regions being left considering the direct detection constraints on the model coefficients.

\subsection{Higgs indirect search}

The current status of the LHC measurements of the Higgs couplings constrains the matrix element $R_{11}$, which describes the discrepancy between $h_{1}$ and the SM Higgs. The couplings of $h_{1}$ to all SM particles are the rescaled values of the SM couplings, taking the form of

$$
g_{h_{1} X X}=R_{11} g_{h_{1} X X}^{S M} .
$$

For the first two group parameter spaces in the last section, additional decay channel $h_{1} \rightarrow A A(V V)$ exists, which almost does not change the total width, since we have the square of $R_{12}\left(R_{13}\right)$ to suppress the magnitude of $\Gamma\left(h_{1} \rightarrow A A(V V)\right)$. The same logic applies to the analysis with the third parameter group used in the last section. ${ }^{4}$ Thus signal rates $\mu_{X X}$ associated with Higgs measurements are functions of $R_{11}$

$$
\mu_{X X}=\frac{\sigma \cdot B R}{\sigma^{S M} \cdot B R^{S M}}=R_{11}^{2},
$$

\footnotetext{
${ }^{4}$ Because the mass of $h_{2}\left(h_{3}\right)$ is above the threshold, additional channel $h_{1} \rightarrow h_{2} h_{2}\left(h_{3} h_{3}\right)$, which changes the total width, does not open. For the study of one Higgs decays to two other Higgs bosons, we refer to [63].
} 

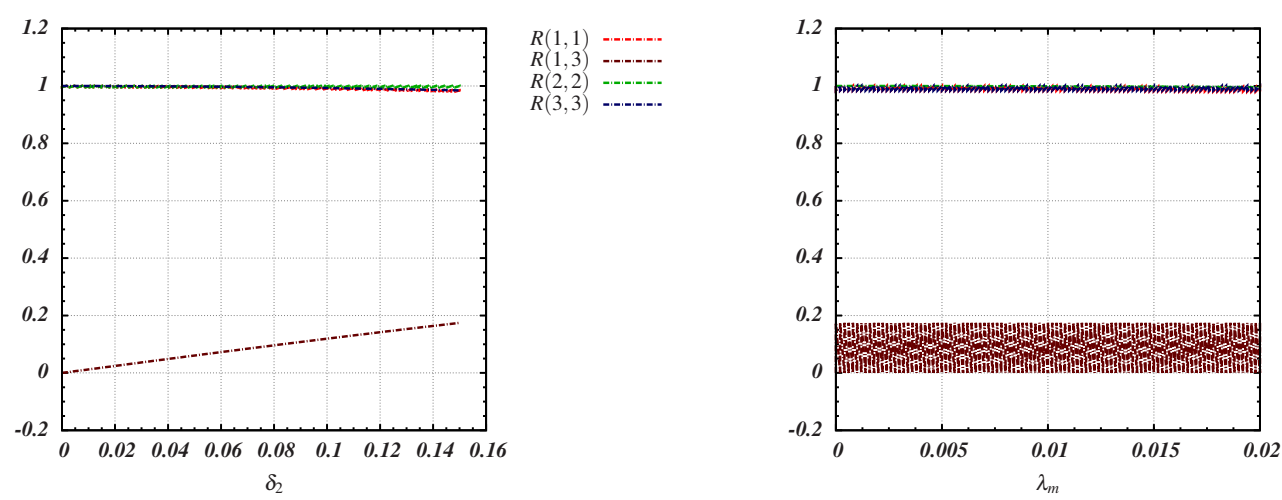

Figure 12. $R$ values with respect to $\lambda_{m}$ and $\delta_{2}$.

with $\sigma, B R$ (that with a a superscript $S M$ ) being the production cross section and branching ratios of $h_{1}$ (the SM Higgs). Therefore, to what extent the model differs from the SM Higgs measurements is determined by the value of $R_{11}$. The value of $R_{11}$ for the parameter setup given in table. 1 is 0.9973 , and we refer to figure 12 for the $R$ vales $^{5}$ corresponding to the benchmark scenario given in table. 2 .

\subsection{Electroweak precision observables constraints}

The presence of the scalar $h_{3}$ with mass $m_{h_{3}}<114 \mathrm{GeV}$ is subject to tight bounds from the LEP [62], while these bounds are highly released in all our parameter choices, since the mixing between $h_{1}$ and $h_{3}$ is very small, about 0.0573 for benchmark scenario given in table. 1 and could be seen in the figure 12 for the benchmark scenario given in table. 2 .

As for electroweak precision observables, the oblique parameters $S$ and $T$ which parameterize potential new physics contributions to electroweak radiative corrections, are computed following refs. [51, 64]. In our model, they are

$$
\begin{aligned}
S=\frac{1}{24 \pi} & \left\{R_{11}^{2}\left[\log R_{h_{1} h}+G\left(m_{h_{1}}^{2}, m_{Z}^{2}\right)-G\left(m_{h_{1}}^{2}, m_{Z}^{2}\right)\right]\right. \\
& +R_{12}^{2}\left[\log R_{h_{2} h}+G\left(m_{h_{2}}^{2}, m_{Z}^{2}\right)-G\left(m_{h_{2}}^{2}, m_{Z}^{2}\right)\right] \\
& \left.+R_{13}^{2}\left[\log R_{h_{3} h}+G\left(m_{h_{3}}^{2}, m_{Z}^{2}\right)-G\left(m_{h_{3}}^{2}, m_{Z}^{2}\right)\right]\right\}, \\
T=\frac{3}{16} & \sin ^{2} \theta_{W}\left\{R_{11}^{2}\left[\frac{1}{\cos ^{2} \theta_{W}}\left(\frac{\log R_{Z h_{1}}}{1-R_{Z h_{1}}}-\frac{\log R_{Z h}}{1-R_{Z h}}\right)-\left(\frac{\log R_{W h_{1}}}{1-R_{W h_{1}}}-\frac{\log R_{W h}}{1-R_{W h}}\right)\right]\right. \\
& +R_{12}^{2}\left[\frac{1}{\cos ^{2} \theta_{W}}\left(\frac{\log R_{Z h_{2}}}{1-R_{Z h_{2}}}-\frac{\log R_{Z h}}{1-R_{Z h}}\right)-\left(\frac{\log R_{W h_{2}}}{1-R_{W h_{2}}}-\frac{\log R_{W h}}{1-R_{W h}}\right)\right] \\
& \left.+R_{13}^{2}\left[\frac{1}{\cos ^{2} \theta_{W}}\left(\frac{\log R_{Z h_{3}}}{1-R_{Z h_{3}}}-\frac{\log R_{Z h}}{1-R_{Z h}}\right)-\left(\frac{\log R_{W h_{3}}}{1-R_{W h_{3}}}-\frac{\log R_{W h}}{1-R_{W h}}\right)\right]\right\},
\end{aligned}
$$

\footnotetext{
${ }^{5}$ Here, we use $R(i, j)$ as the labels in the two plots, which means the same as that of $R_{i j}$ in other place of this work.
} 

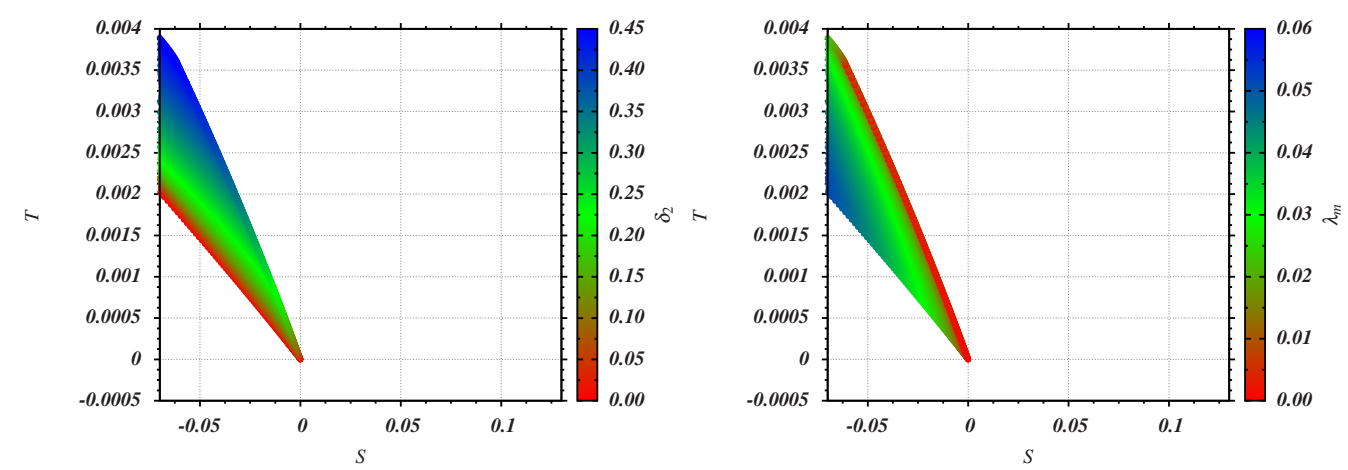

Figure 13. Constraints on $\delta_{2}, \lambda_{m}$ from limits of $\mathrm{S}$ and $\mathrm{T}$.

where, $R_{A B}, G\left(m_{A}^{2}, m_{B}^{2}\right)$ and $f\left(R_{A B}\right)$ are given by

$$
\begin{aligned}
R_{A B}= & \frac{m_{A}^{2}}{m_{B}^{2}}, \\
G\left(m_{A}^{2}, m_{B}^{2}\right)= & -\frac{79}{3}+9 R_{A B}-2 R_{A B}^{2}+\left(12-4 R_{A B}+R_{A B}^{2}\right) f\left(R_{A B}\right) \\
& +\left(-10+18 R_{A B}-6 R_{A B}^{2}+R_{A B}^{3}-9 \frac{R_{A B}+1}{R_{A B}-1}\right) \log R_{A B}, \\
f\left(R_{A B}\right)= & \left\{\begin{array}{cc}
\sqrt{R_{A B}\left(R_{A B}-4\right)} \log \left|\frac{R_{A B}-2-\sqrt{R_{A B}\left(R_{A B}-4\right)}}{2}\right| & R_{A B}>4, \\
0 & R_{A B}=4, \\
2 \sqrt{R_{A B}\left(4-R_{A B}\right)} \arctan \sqrt{\frac{4-R_{A B}}{R_{A B}}} & R_{A B}<4 .
\end{array}\right.
\end{aligned}
$$

The parameters set is given in table 2 and the magnitude of $g_{\phi}$ is determined by $m_{V}$ and $v_{\phi}$. The constraints on two parameters $\delta_{2}$ and $\lambda_{m}$ from $S$ and $T$ parameters are shown in figure 13. The $S$ and $T$ constraints require that $\delta_{2}$ and $\lambda_{m}$ should be smaller than about 0.45 and 0.06 respectively. $S$ and $T$ are very sensitive to the mixing effects. The first two parameter groups (as given in section 3.1) does not change the mixing angles among $h, \eta^{\prime}, S$, and $S$ and $T$ give rise to the null limits since the mixing effects among $h$ and $\eta^{\prime}, S$ in that cases are very small.

\section{Vacuum stability}

The global minimum of the tree-level potential of our model requires,

$$
\lambda>0, \lambda_{\phi}>0, d_{2}>0, \lambda d_{2}>4 \delta_{2}^{2}, \lambda_{\phi} d_{2}>\delta_{1}^{2}, \lambda \lambda_{\phi}>16 \lambda_{m}^{2} .
$$

From one-loop renormalization group equations (RGEs) of the Higgs quartic couplings in appendix C, i.e., eq. (C.1), we find that the Higgs portals couplings $\lambda_{m}$ and $\delta_{2}$ are all get involved and give rise to positive contributions to $\beta_{\lambda}$. Thus, we could expect the vacuum stability problem being solved or alleviated in some parameter spaces. Adopting the central values of top quark mass, the Higgs mass, and the strong coupling [59] as the 

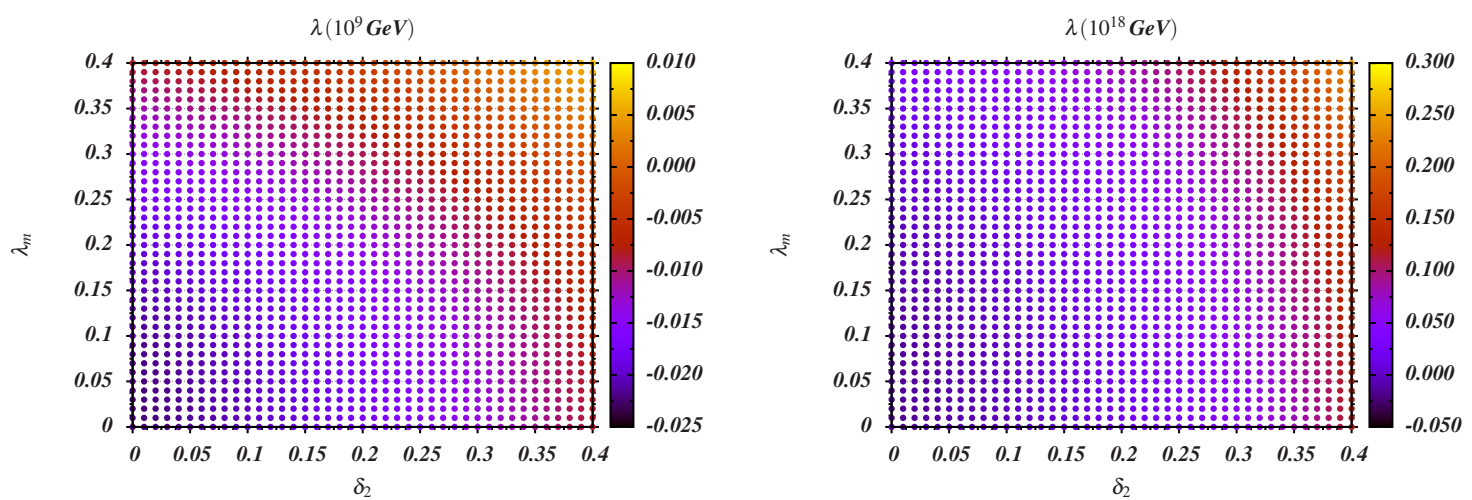

Figure 14. The Higgs quartic coupling $\lambda$ as a function of $\lambda_{m}$ and $\delta_{2}$ at $10^{9} \mathrm{GeV}$ (left panel) and $10^{18} \mathrm{GeV}$ (right panel). With parameters set as: $\lambda=0.129, \lambda_{\phi}=0.2, d_{2}=0.2, \delta_{1}=0.2, \delta_{2}=0.2$, $\lambda_{m}=0.2$.

low energy boundary conditions, we find that with the increasing energy scale, the Higgs quartic coupling running to a negative value around the scale $10^{9} \mathrm{GeV}$, and then grows to positive values latter $[65,66]$.

Based on arguments on vacuum stability given in ref. [66], one needs have positive value of the Higgs quartic coupling to ensure absolute stability. It is obvious that to obtain absolute stability, we should elevate the curves of the Higgs quartic coupling in the plot of $\lambda-\mu[65,66]$. From the $\beta$ functions given in eq. (C.1), we find that with the increasing of $\lambda_{m}$ and $\delta_{2}$ one could have the increasing of the value of the Higgs quartic coupling $\lambda$. To verify this, we explore parameter spaces that survive under $S$ and $T$ limits as shown in figure 14, and we find that $\lambda_{m}$ and $\delta_{2}$ are required to be bigger than around 0.35 in order to evade the stability problem.

To figure out to what extent we need to lift the value of the Higgs quartic coupling in the third parameters setup (as given in table 2), we plot contours of $\lambda\left(\mu=10^{9}\left(10^{18}\right)\right.$ $\mathrm{GeV}$ ) with respect to $\lambda_{m}$ and $\delta_{2}$, as shown in figure 15 , and we find that the vacuum is not bound from bellow there. The plot of $\lambda$ (at the scale of $10^{9} \mathrm{GeV}$ ) as a function of $\lambda_{m}$ and $\delta_{2}$, for the scenario with two $\mathbb{S}$ being supplemented (both two $\mathbb{S}$ have the same interaction with the $\mathrm{SM}$ and the $\mathrm{SU}(2)_{D}$ group), shows that with $\delta_{2}>0.25$ one can indeed obtain the potential bounded from below, as shown in figure 16. The two figures, i.e., figure 15 and figure 16 , illustrate that to achieve the vacuum stability, no smaller than two $\mathbb{S}$ is expected in the third parameters setup.

\section{$6 \quad$ Footprint of the naturalness problem}

In this section, we would like to present the relevant naturalness problem in our model, and discuss the indirect search of the scenario which could improve the naturalness problem. 

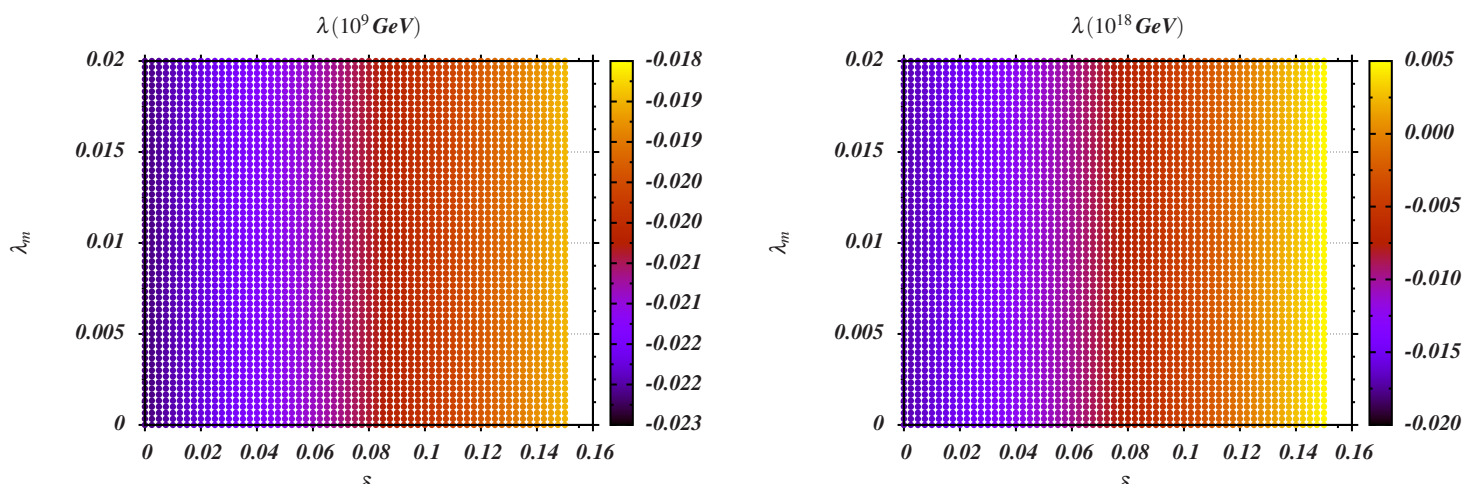

$\delta_{2}$

$\delta_{2}$

Figure 15. The Higgs quartic coupling $\lambda$ as a function of $\lambda_{m}$ and $\delta_{2}$ at $10^{9} \mathrm{GeV}$ (left panel) and $10^{18} \mathrm{GeV}$ (right panel). With parameters set as: $\lambda=0.515, \lambda_{\phi}=0.2, d_{2}=0.2, \delta_{1}=0.02, \delta_{2}=0.04$, $\lambda_{m}=0.01$.

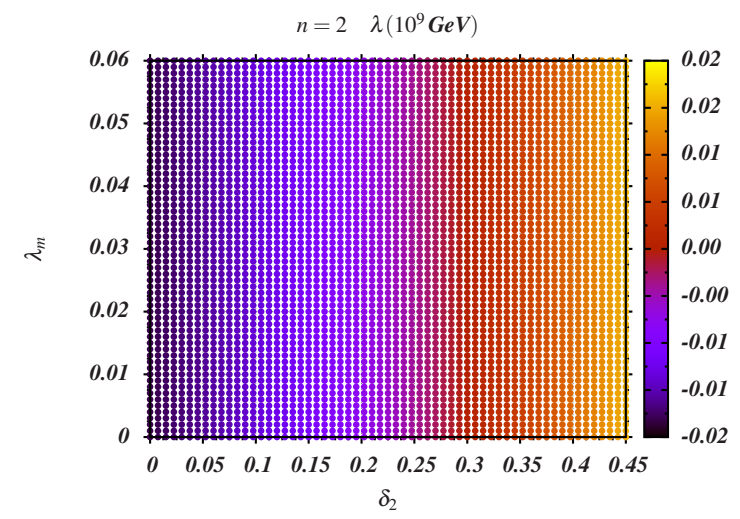

Figure 16. $\lambda\left(10^{9} \mathrm{GeV}\right)$ as a function of $\delta_{2}$ and $\lambda_{m} \cdot n=2$ is to indicate we have supplemented two $\mathbb{S}$.

\subsection{The naturalness problem}

In the SM, considering the gauge invariant property of the two point Higgs Green function [67], the naturalness problem could be defined as

$$
\left(m_{h}^{0}\right)^{2}=m_{h}^{2}+\frac{\Lambda^{2}}{(4 \pi)^{2}} V C_{S M},
$$

where

$$
V C_{S M}=12 \lambda-12 g_{t}+\frac{9}{2} g_{2}^{2}+\frac{3}{2} g_{1}^{2},
$$

and $m_{h}\left(m_{h}^{0}\right)$ is the renormalized (bare) Higgs mass, $\Lambda$ indicates the cut off scale where the new fields are required to cancel the quadratic divergences of the Higgs mass square.

Although our model is renormalizable, we assume it as a low energy effective theory of some more fundamental theory, which is UV completed at the scale $\Lambda$. Thus, eq. (6.2) 
becomes

$$
\left(m_{h}^{0}\right)^{2}=m_{h}^{2}+\frac{\Lambda^{2}}{(4 \pi)^{2}} V C_{V A},
$$

with

$$
\begin{aligned}
V C_{V A} \approx & 12 \lambda+\frac{9}{2} g_{2}^{2}+\frac{3}{2} g_{1}^{2}-12 g_{t}^{2} \\
& +\frac{5}{2} \lambda_{m}+\frac{\lambda_{m}^{2}}{\lambda_{\phi}}+\frac{3 g_{\phi}^{2} \lambda_{m}}{2 \lambda_{\phi}}+n\left(\frac{3}{8} \delta_{2}+\frac{\delta_{2} \lambda_{m}}{\lambda_{\phi}}+\frac{\delta_{2}^{2} d_{2}}{8}+\frac{\delta_{2} \delta_{1}}{8 d_{2}}\right),
\end{aligned}
$$

in which new fields get involved. Here, we suppose that not only one $\mathbb{S}$ embracing the same property as explored in the model (thus $n$ is introduced to denote the number of $\mathbb{S}$ ), and the symbol $\approx$ is to indicate that eq. (6.3) could be hold in the case where $S, \eta^{\prime}$, and $H$ have negligible mixing.

In addition, we would like to mention that, the VEV and Higgs mass in the SM are gauge invariant considering the renormalization of the mass term in the SM Higgs potential [68]. And [68] use the different tadpole renormalization method in comparison with that of [67] and leaves no trails of tadpole contributions in $V C_{S M}$ and $V C_{V A}$, which cast the new formula of

$$
\begin{aligned}
& V C_{S M}^{\prime}=-\left(6 \lambda-6 g_{t}+\frac{9}{4} g_{2}^{2}+\frac{3}{4} g_{1}^{2}\right) \\
& V C_{V A}^{\prime} \approx-\left(6 \lambda+\frac{9}{4} g_{2}^{2}+\frac{3}{4} g_{1}^{2}-6 g_{t}^{2}\right)-\frac{1}{2} \lambda_{m}-\frac{n}{2} \delta_{2} .
\end{aligned}
$$

To soften the naturalness problem [4,5], the simplest solution is to suppose $V C_{V A}=0$ (or $V C_{V A}^{\prime}=0$ ) so that the modified Veltman condition [69] is realized. For $\Lambda=10 \mathrm{TeV}$, $n \approx 1(6)$ is needed to obtain $V C_{V A}=0\left(V C_{V A}^{\prime}=0\right)$.

\subsection{Indirect search for the scenario which alleviates the naturalness problem}

Considering $m_{\eta^{\prime}, S, A} \gg v$ could been satisfied in some parameter spaces of the model, ${ }^{6}$ one may integrate out the $\eta^{\prime}, S, A$ and express their effects in terms of an effective Lagrangian below the scale $\operatorname{Min}\left(m_{\eta^{\prime}}, m_{S}, m_{A}\right)$, which involves only the SM fields with appropriate higher-dimensional operators. At one-loop level, integrating out the $\eta^{\prime}, S, A$ leads to shifts in the wave-function renormalization and the potential of the Higgs doublet $H$, as well as operators with dimension six and higher. The dimension-six operators in the effective Lagrangian cast the form of

$$
\mathcal{L}_{\text {eff }}=\mathcal{L}_{S M}+\left(\frac{c_{H}^{\eta^{\prime}}}{m_{\eta^{\prime}}^{2}}+\frac{c_{H}^{s}}{m_{S}^{2}}+\frac{c_{H}^{A}}{m_{A}^{2}}\right)\left(\frac{1}{2} \partial_{\mu}|H|^{2} \partial^{\mu}|H|^{2}\right)
$$

\footnotetext{
${ }^{6}$ For simplicity, we consider the case in which the mixings among the three fields $\eta^{\prime}, S, A$ could be neglected.
} 
Matching to the full theory at the scale $m_{\eta^{\prime}, s, A}$, we have

$$
\begin{aligned}
c_{H}^{\eta^{\prime}} & =\frac{n_{\eta^{\prime}}}{4}\left|\lambda_{m}\right|^{2} /\left(96 \pi^{2}\right), \\
c_{H}^{S} & =\frac{n}{8}\left|\delta_{2}\right|^{2} /\left(96 \pi^{2}\right), \\
c_{H}^{A} & =\frac{n}{8}\left|\delta_{2}\right|^{2} /\left(96 \pi^{2}\right) .
\end{aligned}
$$

Below the scale of electroweak symmetry breaking, eq. (6.6) leads to a shift in the wavefunction renormalization of the physical scalar $h$ [9], with $\delta Z_{h}=2 v^{2}\left(c_{H}^{\eta^{\prime}} / m_{\eta^{\prime}}^{2}+c_{H}^{S} / m_{S}^{2}+\right.$ $\left.c_{H}^{A} / m_{A}^{2}\right)$. After canonically normalizing $h$, i.e., $h \rightarrow\left(1-\delta Z_{h} / 2\right) h$, its coupling to vectors and fermions are altered, which may lead to a measurable correction to, e.g., the $h Z$ associated production cross-section

$$
\delta \sigma_{Z h}=-2 v^{2}\left(c_{H}^{\eta^{\prime}} / m_{\eta}^{\prime 2}+c_{H}^{S} / m_{S}^{2}+c_{H}^{A} / m_{A}^{2}\right),
$$

where we have defined $\delta \sigma_{Z h}$ as the fractional change in the associated production cross section relative to the SM prediction, which by design vanishes for the case of the SM. ${ }^{7}$ The sizable $n_{\eta^{\prime}}(n)$, being required to relax the naturalness problem ${ }^{8}$ (see eq. (6.3)), may causes the correspondingly observable effect in the precision measurement of $\sigma_{Z h}$, which is what the future lepton colliders supposed to detect. At last, if one use our model to analyze electroweak phase transition, the measurement of associated production cross section of $Z h$ $\left(\sigma_{Z h}\right)$ might imposes very strong constraint on the multi-Higgs portals couplings $\lambda_{m}$ and $\delta_{2}$.

\section{Discussions and conclusion}

In our model, the vector field $(V)$ and the imaginary part of complex singlet $(A)$ are stable due to the reduced custodial symmetry $\mathrm{SO}(3)$ and the residual $Z_{2}$ symmetry. The interactions between the two DMs and resonant effects mediated by three Higgs portals are demonstrated through the study of the relic density behavior with respect to $m_{A}$ and $m_{V}$. The effects of the multi-Higgs portals couplings on the generation of DM relic density are shown in the parameter space of $\lambda_{m}$ and $\delta_{2}$. The parameter spaces of $\lambda_{m}$ and $\delta_{2}$ are totally free under the LUX experiment results limits. Constraints (from LUX experimental results) on parameter spaces of $m_{A}-m_{V}$ give rise to $m_{A}>30 \mathrm{GeV}\left(m_{A}\left(m_{V}\right)>50 \mathrm{GeV}\right)$ for the case of $m_{A}>m_{V}\left(m_{A}<m_{V}\right)$, which allows more relaxed parameter spaces in compare with the simple models. As for the third parameter group: the electroweak precision observables require $\lambda_{m}<0.06$ and $\delta_{2}<0.45$, the vacuum stability at high scale requires at least two complex singlet, which is supposed to be consistent with the naturalness problem argument. Other benchmark scenario with one $\mathbb{S}$, which survives under indirect Higgs search and electroweak precision test limits, and could improve the stability problem is also explored.

\footnotetext{
${ }^{7}$ Here, we would like to mention that, which one of $c_{H}^{\eta}, c_{H}^{s}, c_{H}^{A}$ in the our model takes part in $\delta \sigma_{Z h}$ depends on parameter choices, i.e, the derivations of $c_{H}^{\eta^{\prime}}, c_{H}^{S}, c_{H}^{A}$ require $m_{\eta^{\prime}, S, A} \gg v$.

${ }^{8}$ The sizable $n$ is also necessary from the viewpoint of the vacuum stability, see section 5 .
} 
At last, we would like to mention that, through tuning of the parameter $\delta_{2}$ associate with tuning of $v_{s}$ in the first setup of parameter spaces,${ }^{9}$ one could use the model to explain Galactic Center Excess observed by the Fermi Telescope, with $\langle\sigma v\rangle \approx 10^{-26} \mathrm{~cm}^{3} / \mathrm{s}$ being provided by scalar DM pairs annihilating to $b \bar{b}$ and the relic density supplied by the vector DM. ${ }^{10}$ Details of which, and the realization of SFOEWPT and inflation in the model are left for further studies.

\section{A Annihilation cross sections}

When the Higgs mass $m_{h_{i}}$ is larger than twice of the SM particle masses, the corresponding visible decay channels widths are

$$
\begin{aligned}
\Gamma_{h_{i} \rightarrow f f} & =\frac{N_{c} G_{F} m_{f}^{2}}{4 \sqrt{2} \pi} m_{h_{i}}\left|R_{i 1}\right|^{2}\left(1-\frac{4 m_{f}^{2}}{m_{h_{i}}^{2}}\right)^{3 / 2}, \\
\Gamma_{h_{i} \rightarrow W W} & =\frac{G_{F}}{8 \sqrt{2} \pi} m_{h_{i}}^{3}\left|R_{i 1}\right|^{2} \sqrt{1-\frac{4 m_{W}^{2}}{m_{h_{i}}^{2}}}\left(1-\frac{4 m_{W}^{2}}{m_{h_{i}}^{2}}+\frac{12 m_{W}^{4}}{m_{h_{i}}^{4}}\right), \\
\Gamma_{h_{i} \rightarrow Z Z} & =\frac{G_{F}}{16 \sqrt{2} \pi} m_{h_{i}}^{3}\left|R_{i 1}\right|^{2} \sqrt{1-\frac{4 m_{Z}^{2}}{m_{h_{i}}^{2}}}\left(1-\frac{4 m_{Z}^{2}}{m_{h_{i}}^{2}}+\frac{12 m_{Z}^{4}}{m_{h_{i}}^{4}}\right),
\end{aligned}
$$

and when the Higgs mass $m_{h_{i}}$ is larger than the twice of the DM mass, the invisible decay channels widths are

$$
\begin{gathered}
\Gamma_{h_{i} \rightarrow V V}=\left|R_{i 2} g_{\phi}^{2} \frac{v_{\phi}}{v}\right|^{2} \frac{v^{2} m_{h_{i}}^{3}}{128 \pi m_{V}^{4}} \sqrt{1-\frac{4 m_{V}^{2}}{m_{h_{i}}^{2}}}\left(1-\frac{4 m_{V}^{2}}{m_{h_{i}}^{2}}+\frac{12 m_{V}^{4}}{m_{h_{i}}^{4}}\right), \\
\Gamma_{h_{i} \rightarrow A A}=\left|R_{i 1} \frac{\delta_{2}}{2}+R_{i 2} \frac{\delta_{1}}{2} \frac{v_{\phi}}{v}+R_{i 3} \frac{d_{2}}{2} \frac{v_{s}}{v}\right|^{2} \frac{v^{2}}{32 \pi m_{h_{i}}} \sqrt{1-\frac{4 m_{A}^{2}}{m_{h_{i}}^{2}}} .
\end{gathered}
$$

The total decay widthes of the Higgs with mass $m_{h_{i}}$ are the summations of visible and invisible decay widths.

The annihilation cross sections for DM corresponding to figure 1 are given as follows:

\section{- Top-left}

$$
\begin{aligned}
<\sigma v>_{V V \rightarrow h_{j} h_{j}}^{s}= & \frac{1}{32 \pi} \frac{g_{\phi}^{4} v_{\phi}^{2}}{m_{V}^{2}} \sqrt{1-\frac{m_{h_{1}}^{2}}{m_{V}^{2}}} \\
& \times\left|\left[\sum_{i} \frac{R_{2 i}\left(R_{i 1} R_{1 j} \lambda v+R_{i 2} R_{2 j} \lambda_{m} v_{\phi}+R_{i 3} R_{3 j} \frac{\delta_{2}}{2} v_{s}\right)}{4 m_{V}^{2}-m_{h_{i}}^{2}+i m_{h_{i}} \Gamma_{h_{i}}}\right]\right|^{2}, \\
<\sigma v>_{V V \rightarrow f f}= & n_{c} \sum_{f} \frac{m_{f}^{2}}{16 \pi}\left(1-\frac{m_{f}^{2}}{m_{V}^{2}}\right)^{3 / 2}\left|\sum_{i} \frac{\left(g_{\phi}^{2} v_{\phi} / v\right) R_{2 i} R_{i 1}}{4 m_{V}^{2}-m_{h_{i}}^{2}+i m_{h_{i}} \Gamma_{h_{i}}}\right|^{2},
\end{aligned}
$$

\footnotetext{
${ }^{9}$ In order to maintain small mixing between $h$ and $S$ which helps to escape constraints of LHC and electroweak observable experiments.

${ }^{10}$ For a viable annihilating multi-component dark matter model consisting of two real gauge singlet scalars, one can explain the low energy $(1-3 \mathrm{GeV})$ gamma ray excess from both Galactic Centre and Fermi Bubble [71].
} 


$$
\begin{aligned}
& <\sigma v>_{V V \rightarrow Z Z}=\frac{m_{V}^{2}}{128 \pi} \sqrt{1-\frac{m_{Z}^{2}}{m_{V}^{2}}}\left(1-\frac{m_{Z}^{2}}{m_{V}^{2}}+\frac{3 m_{Z}^{4}}{m_{V}^{4}}\right)\left|\sum_{i} \frac{\left(g_{\phi}^{2} v_{\phi} / v\right) R_{2 i} R_{i 1}}{4 m_{V}^{2}-m_{h_{i}}^{2}+i m_{h_{i}} \Gamma_{h_{i}}}\right|^{2}, \\
& <\sigma v>_{V V \rightarrow W W}=\frac{m_{V}^{2}}{64 \pi} \sqrt{1-\frac{m_{W}^{2}}{m_{V}^{2}}}\left(1-\frac{m_{W}^{2}}{m_{V}^{2}}+\frac{3 m_{W}^{4}}{m_{V}^{4}}\right)\left|\sum_{i} \frac{\left(g_{\phi}^{2} v_{\phi} / v\right) R_{2 i} R_{i 1}}{4 m_{V}^{2}-m_{h_{i}}^{2}+i m_{h_{i}} \Gamma_{h_{i}}}\right|^{2}, \\
& <\sigma v>_{A A \rightarrow h_{j} h_{j}}^{s}=\frac{1}{32 \pi} \frac{1}{m_{A}^{2}} \sqrt{1-\frac{m_{h_{1}}^{2}}{m_{A}^{2}}} \\
& \times \mid \frac{\delta_{2}}{2} v\left[\sum_{i} \frac{R_{1 i}\left(R_{i 1} R_{1 j} \lambda v+R_{i 2} R_{2 j} \lambda_{m} v_{\phi}+R_{i 3} R_{3 j} \frac{\delta_{2}}{2} v_{s}\right)}{4 m_{A}^{2}-m_{h_{i}}^{2}+i m_{h_{i}} \Gamma_{h_{i}}}\right] \\
& +\frac{\delta_{1}}{2} v_{\phi}\left[\sum_{i} \frac{R_{2 i}\left(R_{i 1} R_{1 j} \lambda v+R_{i 2} R_{2 j} \lambda_{m} v_{\phi}+R_{i 3} R_{3 j} \frac{\delta_{2}}{2} v_{s}\right)}{4 m_{A}^{2}-m_{h_{i}}^{2}+i m_{h_{i}} \Gamma_{h_{i}}}\right] \\
& +\left.\frac{d_{2}}{2} v_{s}\left[\sum_{i} \frac{R_{3 i}\left(R_{i 1} R_{1 j} \lambda v+R_{i 2} R_{2 j} \lambda_{m} v_{\phi}+R_{i 3} R_{3 j} \frac{\delta_{2}}{2} v_{s}\right)}{4 m_{A}^{2}-m_{h_{i}}^{2}+i m_{h_{i}} \Gamma_{h_{i}}}\right]\right|^{2}, \\
& <\sigma v>_{A A \rightarrow f f}=n_{c} \sum_{f} \frac{m_{f}^{2}}{16 \pi}\left(1-\frac{m_{f}^{2}}{m_{A}^{2}}\right)^{3 / 2} \mid\left[\sum_{i} \frac{\left(\delta_{2} / 2\right) R_{1 i} R_{i 1}}{4 m_{A}^{2}-m_{h_{i}}^{2}+i m_{h_{i}} \Gamma_{h_{i}}}\right] \\
& \times\left[\sum_{i} \frac{\left(\delta_{1} v_{\phi} / 2 v\right) R_{2 i} R_{i 1}}{4 m_{A}^{2}-m_{h_{i}}^{2}+i m_{h_{i}} \Gamma_{h_{i}}}\right]+\left.\left[\sum_{i} \frac{\left(d_{2} v_{s} / 2 v\right) R_{3 i} R_{i 1}}{4 m_{A}^{2}-m_{h_{i}}^{2}+i m_{h_{i}} \Gamma_{h_{i}}}\right]\right|^{2}, \\
& <\sigma v>_{A A \rightarrow Z Z}=\frac{m_{A}^{2}}{128 \pi} \sqrt{1-\frac{m_{Z}^{2}}{m_{A}^{2}}}\left(1-\frac{m_{Z}^{2}}{m_{A}^{2}}+\frac{3 m_{Z}^{4}}{m_{A}^{4}}\right) \times \mid\left[\sum_{i} \frac{\left(\delta_{2} / 2\right) R_{1 i} R_{i 1}}{4 m_{A}^{2}-m_{h_{i}}^{2}+i m_{h_{i}} \Gamma_{h_{i}}}\right] \\
& +\left[\sum_{i} \frac{\left(\delta_{1} v_{\phi} / 2 v\right) R_{2 i} R_{i 1}}{4 m_{A}^{2}-m_{h_{i}}^{2}+i m_{h_{i}} \Gamma_{h_{i}}}\right]+\left.\left[\sum_{i} \frac{\left(d_{2} v_{s} / 2 v\right) R_{3 i} R_{i 1}}{4 m_{A}^{2}-m_{h_{i}}^{2}+i m_{h_{i}} \Gamma_{h_{i}}}\right]\right|^{2}, \\
& <\sigma v>_{A A \rightarrow W W}=\frac{m_{A}^{2}}{64 \pi} \sqrt{1-\frac{m_{W}^{2}}{m_{A}^{2}}}\left(1-\frac{m_{W}^{2}}{m_{A}^{2}}+\frac{3 m_{W}^{4}}{m_{A}^{4}}\right) \times \mid\left[\sum_{i} \frac{\left(\delta_{2} / 2\right) R_{1 i} R_{i 1}}{4 m_{A}^{2}-m_{h_{i}}^{2}+i m_{h_{i}} \Gamma_{h_{i}}}\right] \\
& +\left[\sum_{i} \frac{\left(\delta_{1} v_{\phi} / 2 v\right) R_{2 i} R_{i 1}}{4 m_{A}^{2}-m_{h_{i}}^{2}+i m_{h_{i}} \Gamma_{h_{i}}}\right]+\left.\left[\sum_{i} \frac{\left(d_{2} v_{s} / 2 v\right) R_{3 i} R_{i 1}}{4 m_{A}^{2}-m_{h_{i}}^{2}+i m_{h_{i}} \Gamma_{h_{i}}}\right]\right|^{2} .
\end{aligned}
$$

\section{- Top-right}

$$
\begin{aligned}
& <\sigma v>_{V V \rightarrow A A}=\frac{1}{32 \pi} \frac{1}{m_{V}^{2}} \sqrt{1-\frac{m_{A}^{2}}{m_{V}^{2}}}\left|\left[\sum_{i} \frac{\left(g_{\phi}^{2} v_{\phi}\right) R_{2 i}\left(R_{i 1} \frac{\delta_{2}}{2} v+R_{i 2} \frac{\delta_{1}}{2} v_{\phi}+R_{i 3} \frac{d_{2}}{2} v_{s}\right)}{4 m_{V}^{2}-m_{h_{i}}^{2}+i m_{h_{i}} \Gamma_{h_{i}}}\right]\right|^{2}, \\
& <\sigma v>_{A A \rightarrow V V}=\frac{m_{A}^{2}}{128 \pi} \sqrt{1-\frac{m_{V}^{2}}{m_{A}^{2}}}\left(1-\frac{m_{V}^{2}}{m_{A}^{2}}+\frac{3 m_{V}^{4}}{m_{A}^{4}}\right) \times \mid\left[\sum_{i} \frac{\left(\delta_{2} / 2\right) R_{1 i} R_{i 1}}{4 m_{A}^{2}-m_{h_{i}}^{2}+i m_{h_{i}} \Gamma_{h_{i}}}\right]
\end{aligned}
$$




$$
+\left[\sum_{i} \frac{\left(\delta_{1} v_{\phi} / 2 v\right) R_{2 i} R_{i 1}}{4 m_{A}^{2}-m_{h_{i}}^{2}+i m_{h_{i}} \Gamma_{h_{i}}}\right]+\left.\left[\sum_{i} \frac{\left(d_{2} v_{s} / 2 v\right) R_{3 i} R_{i 1}}{4 m_{A}^{2}-m_{h_{i}}^{2}+i m_{h_{i}} \Gamma_{h_{i}}}\right]\right|^{2} .
$$

- Bottom-left

$$
\begin{aligned}
& <\sigma v>_{V V \rightarrow h_{i} h_{i}}^{t+u}=\frac{1}{4 \pi} \frac{1}{m_{V}^{2}} \sqrt{1-\frac{m_{h_{i}}^{2}}{m_{V}^{2}}}\left|\frac{\left(g_{\phi}^{2} v_{\phi}\right)^{2}}{m_{h_{i}}^{2}-m_{V}^{2}}\right|^{2} R_{2 i}^{2} \\
& <\sigma v>_{A A \rightarrow h_{i} h_{i}}^{t+u}=\frac{1}{4 \pi} \frac{1}{m_{A}^{2}}\left[\sqrt{1-\frac{m_{h_{1}}^{2}}{m_{A}^{2}}}\left|\frac{\left(\delta_{2} v / 2\right)^{2} R_{1 i}}{m_{h_{1}}^{2}-m_{A}^{2}}\right|^{2}+\sqrt{1-\frac{m_{h_{2}}^{2}}{m_{A}^{2}}}\left|\frac{\left(\delta_{1} v_{\phi} / 2\right)^{2} R_{2 i}}{m_{h_{2}}^{2}-m_{A}^{2}}\right|^{2}\right. \\
& \left.+\sqrt{1-\frac{m_{h_{3}}^{2}}{m_{A}^{2}}}\left|\frac{\left(d_{2} v_{s} / 2\right)^{2} R_{3 i}}{m_{h_{3}}^{2}-m_{A}^{2}}\right|^{2}\right] \text {. }
\end{aligned}
$$

- Bottom-right

$$
\begin{aligned}
<\sigma v>_{V V \rightarrow h_{i} h_{i}}^{\text {seagull }}= & \frac{1}{32 \pi} \frac{1}{m_{V}^{2}} \sqrt{1-\frac{m_{h_{i}}^{2}}{m_{V}^{2}}}\left|\frac{g_{\phi}^{2}}{2}\right|^{2} R_{2 i}^{2}, \\
\left\langle\sigma v>_{\substack{\text { sA } \rightarrow h_{i} h_{i} \\
\text { seagull }}}\right. & \frac{1}{32 \pi} \frac{1}{m_{A}^{2}}\left[\sqrt{1-\frac{m_{h_{1}}^{2}}{m_{A}^{2}}}\left|\frac{\delta_{2}}{2}\right|^{2} R_{1 i}^{2}+\sqrt{1-\frac{m_{h_{2}}^{2}}{m_{A}^{2}}}\left|\frac{\delta_{1}}{2} \frac{v_{\phi}}{v}\right|^{2} R_{2 i}^{2}\right. \\
& \left.+\sqrt{1-\frac{m_{h_{3}}^{2}}{m_{A}^{2}}}\left|\frac{d_{2}}{2} \frac{v_{s}}{v}\right|^{2} R_{3 i}^{2}\right] .
\end{aligned}
$$

\section{B Boltzman equations}

The coupled boltzman equations could be written as [3]:

$$
\begin{aligned}
\frac{d Y_{A}}{d x_{A}}= & -\frac{1.32 g_{\star}^{1 / 2} M_{A} M_{p}}{x_{A}^{2}}\left(\left\langle\sigma v_{r e l}\right\rangle_{A A \rightarrow X \bar{X}}\left(Y_{A}^{2}-\left(Y_{A}^{e q}\right)^{2}\right)\right. \\
& \left.+\left\langle\sigma v_{r e l}\right\rangle_{A A \rightarrow V V}\left(Y_{A}^{2}-\frac{\left(Y_{A}^{e q}\right)^{2}}{\left(Y_{V}^{e q}\right)^{2}} Y_{V}^{2}\right)\right), \\
\frac{d Y_{V}}{d x_{V}}= & -\frac{1.32 g_{\star}^{1 / 2} M_{V} M_{p}}{x_{V}^{2}}\left(\left\langle\sigma v_{r e l}\right\rangle_{V V \rightarrow X \bar{X}}\left(Y_{V}^{2}-\left(Y_{V}^{e q}\right)^{2}\right)\right. \\
& \left.-\left\langle\sigma v_{r e l}\right\rangle_{A A \rightarrow V V}\left(Y_{A}^{2}-\frac{\left(Y_{A}^{e q}\right)^{2}}{\left(Y_{V}^{e q}\right)^{2}} Y_{V}^{2}\right)\right),
\end{aligned}
$$

for $M_{A}>M_{V}$. 
Similarly, for $M_{V}>M_{A}$, one has

$$
\begin{aligned}
\frac{d Y_{V}}{d x_{V}}= & -\frac{1.32 g_{\star}^{1 / 2} M_{V} M_{p}}{x_{V}^{2}}\left(\left\langle\sigma v_{r e l}\right\rangle_{V V \rightarrow X \bar{X}}\left(Y_{V}^{2}-\left(Y_{V}^{e q}\right)^{2}\right)\right. \\
& \left.+\left\langle\sigma v_{r e l}\right\rangle_{V V \rightarrow A A}\left(Y_{V}^{2}-\frac{\left(Y_{V}^{e q}\right)^{2}}{\left(Y_{A}^{e q}\right)^{2}} Y_{A}^{2}\right)\right), \\
\frac{d Y_{A}}{d x_{A}}= & -\frac{1.32 g_{\star}^{1 / 2} M_{A} M_{p}}{x_{A}^{2}}\left(\left\langle\sigma v_{r e l}\right\rangle_{A A \rightarrow X \bar{X}}\left(Y_{A}^{2}-\left(Y_{A}^{e q}\right)^{2}\right)\right. \\
& \left.-\left\langle\sigma v_{r e l}\right\rangle_{V V \rightarrow A A}\left(Y_{V}^{2}-\frac{\left(Y_{V}^{e q}\right)^{2}}{\left(Y_{A}^{e q}\right)^{2}} Y_{A}^{2}\right)\right) .
\end{aligned}
$$

Here, $M_{p}=2.44 \times 10^{18} \mathrm{GeV}$ is the reduced Planck mass, and $g_{\star}$ is the degrees of freedom parameter. Two dimensionless variables $Y_{i, j}$ relate with number density through $Y_{i, j}=\frac{n_{i, j}}{s}$, $x_{i, j}=\frac{m_{i, j}}{T}[70]$, and $s$ and $T$ are the entropy density and temperature of the Universe. After solving the coupled eqs. (B.1), (B.2), (B.3), and (B.4), one gets the values of $Y_{A}$ and $Y_{V}$ at present temperature $T_{0}$.

\section{One loop $\beta$ functions}

The one-loop renormalization group equations (RGEs), which we used to analyze the vacuum stability problem, are given by

$$
\frac{d X}{d \log \mu}=\frac{1}{16 \pi^{2}} \beta_{X}
$$

with one-loop $\beta$-functions $\beta_{X}$,

$$
\begin{aligned}
\beta_{\delta_{1}} & =\frac{1}{2}\left(4 d_{2} \delta_{1}-9 g_{\phi}^{2} \delta_{1}^{2}+4 \delta_{1}^{2}+3 \delta_{1} \lambda_{\phi}+2 \lambda_{m} \delta_{2}\right) \\
\beta_{\delta_{2}} & =\frac{1}{2}\left(4 d_{2} \delta_{2}-3 g_{1}^{2} \delta_{2}-9 g_{2}^{2} \delta_{2}+12 g_{t}^{2} \delta_{2}+4 \delta_{2}^{2}+12 \delta_{2} \lambda+2 \delta_{1} \lambda_{m}\right) \\
\beta_{d_{2}} & =\frac{1}{2}\left(10 d_{2}^{2}+\delta_{1}^{2}+\delta_{2}^{2}\right) \\
\beta_{\lambda_{m}} & =\frac{1}{2}\left(\delta_{1} \delta_{2}-3 g_{1}^{2} \lambda_{m}-9 g_{2}^{2} \lambda_{m}+12 g_{t}^{2} \lambda_{m}-9 g_{\phi}^{2} \lambda_{m}+12 \lambda \lambda_{m}+8 \lambda_{m}^{2}+3 \lambda_{m} \lambda_{\phi}\right) \\
\beta_{\lambda} & =-\lambda\left(3 g_{1}^{2}+9 g_{2}^{2}-12 g_{t}^{2}-24 \lambda\right)+\frac{3}{4} g_{2}^{4}+\frac{3}{8}\left(g_{1}^{2}+g_{2}^{2}\right)^{2}-6 g_{t}^{4}+\frac{\delta_{2}^{2}}{4}+\frac{\lambda_{m}^{2}}{2} \\
\beta_{\lambda_{\phi}} & =\frac{1}{2}\left(9 g_{\phi}^{4}+2 \delta_{1}^{2}+4 \lambda_{m}^{2}-18 g_{\phi}^{2} \lambda_{\phi}+9 \lambda_{\phi}^{2}\right) \\
\beta_{g_{\phi}} & =-\frac{43}{6} g_{\phi}^{3}
\end{aligned}
$$

\section{Acknowledgments}

LGB thank Michael Ramsey-Musuolf, Ran Ding and Bin Zhu for useful discussions. This research was supported in part by the Natural Science Foundation of China under grant numbers 10821504, 11075194, 11135003, 11275246, and 11475238, and by the National Basic Research Program of China (973 Program) under grant number 2010CB833000. 
Open Access. This article is distributed under the terms of the Creative Commons Attribution License (CC-BY 4.0), which permits any use, distribution and reproduction in any medium, provided the original author(s) and source are credited.

\section{References}

[1] ATLAS collaboration, Observation of a new particle in the search for the Standard Model Higgs boson with the ATLAS detector at the LHC, Phys. Lett. B 716 (2012) 1 [arXiv: 1207.7214] [INSPIRE].

[2] CMS collaboration, Observation of a new boson at a mass of $125 \mathrm{GeV}$ with the CMS experiment at the LHC, Phys. Lett. B 716 (2012) 30 [arXiv:1207.7235] [INSPIRE].

[3] L. Bian, R. Ding and B. Zhu, Two Component Higgs-Portal Dark Matter, Phys. Lett. B 728 (2014) 105 [arXiv: 1308.3851] [INSPIRE].

[4] I. Chakraborty and A. Kundu, Controlling the fine-tuning problem with singlet scalar dark matter, Phys. Rev. D 87 (2013) 055015 [arXiv: 1212.0394] [INSPIRE].

[5] B. Grzadkowski and J. Wudka, Pragmatic approach to the little hierarchy problem: the case for Dark Matter and neutrino physics, Phys. Rev. Lett. 103 (2009) 091802 [arXiv:0902.0628] [INSPIRE].

[6] C.N. Karahan and B. Korutlu, Effects of a Real Singlet Scalar on Veltman Condition, Phys. Lett. B 732 (2014) 320 [arXiv: 1404.0175] [InSPIRE].

[7] O. Antipin, M. Mojaza and F. Sannino, Conformal Extensions of the Standard Model with Veltman Conditions, Phys. Rev. D 89 (2014) 085015 [arXiv: 1310.0957] [inSPIRE].

[8] B. Henning, X. Lu and H. Murayama, What do precision Higgs measurements buy us?, arXiv:1404.1058 [INSPIRE].

[9] N. Craig, C. Englert and M. McCullough, New Probe of Naturalness, Phys. Rev. Lett. 111 (2013) 121803 [arXiv:1305.5251] [INSPIRE].

[10] C. Englert and M. McCullough, Modified Higgs Sectors and NLO Associated Production, JHEP 07 (2013) 168 [arXiv: 1303.1526] [INSPIRE].

[11] M. Farina, M. Perelstein and N. Rey-Le Lorier, Higgs Couplings and Naturalness, Phys. Rev. D 90 (2014) 015014 [arXiv: 1305.6068] [INSPIRE].

[12] A. Noble and M. Perelstein, Higgs self-coupling as a probe of electroweak phase transition, Phys. Rev. D 78 (2008) 063518 [arXiv:0711.3018] [INSPIRE].

[13] P.H. Damgaard, D. O'Connell, T.C. Petersen and A. Tranberg, Constraints on New Physics from Baryogenesis and Large Hadron Collider Data, Phys. Rev. Lett. 111 (2013) 221804 [arXiv: 1305.4362] [INSPIRE].

[14] S. Profumo, M.J. Ramsey-Musolf, C.L. Wainwright and P. Winslow, Singlet-catalyzed electroweak phase transitions and precision Higgs boson studies, Phys. Rev. D 91 (2015) 035018 [arXiv: 1407 .5342] [INSPIRE].

[15] H. Davoudiasl, I. Lewis and E. Ponton, Electroweak Phase Transition, Higgs Diphoton Rate and New Heavy Fermions, Phys. Rev. D 87 (2013) 093001 [arXiv:1211.3449] [INSPIRE].

[16] M. Carena, A. Megevand, M. Quirós and C.E.M. Wagner, Electroweak baryogenesis and new TeV fermions, Nucl. Phys. B 716 (2005) 319 [hep-ph/0410352] [INSPIRE]. 
[17] M. Gonderinger, Y. Li, H. Patel and M.J. Ramsey-Musolf, Vacuum Stability, Perturbativity and Scalar Singlet Dark Matter, JHEP 01 (2010) 053 [arXiv:0910.3167] [INSPIRE].

[18] A. Drozd, B. Grzadkowski and J. Wudka, Multi-Scalar-Singlet Extension of the Standard Model - the Case for Dark Matter and an Invisible Higgs Boson, JHEP 04 (2012) 006 [Erratum ibid. 1411 (2014) 130] [arXiv:1112.2582] [INSPIRE].

[19] S. Baek, P. Ko, W.-I. Park and E. Senaha, Higgs Portal Vector Dark Matter: Revisited, JHEP 05 (2013) 036 [arXiv:1212.2131] [INSPIRE].

[20] S. Baek, P. Ko and W.-I. Park, Singlet Portal Extensions of the Standard Seesaw Models to a Dark Sector with Local Dark Symmetry, JHEP 07 (2013) 013 [arXiv:1303.4280] [INSPIRE].

[21] E. Gabrielli et al., Towards Completing the Standard Model: Vacuum Stability, EWSB and Dark Matter, Phys. Rev. D 89 (2014) 015017 [arXiv: 1309.6632] [INSPIRE].

[22] T. Hambye and A. Strumia, Dynamical generation of the weak and Dark Matter scale, Phys. Rev. D 88 (2013) 055022 [arXiv: 1306.2329] [INSPIRE].

[23] P. Ko and W.-I. Park, Higgs-portal assisted Higgs inflation with a large tensor-to-scalar ratio, arXiv:1405.1635 [INSPIRE].

[24] N. Haba and R. Takahashi, Higgs inflation with singlet scalar dark matter and right-handed neutrino in light of BICEP2, Phys. Rev. D 89 (2014) 115009 [arXiv:1404.4737] [InSPIRE].

[25] G. Duda, G. Gelmini and P. Gondolo, Detection of a subdominant density component of cold dark matter, Phys. Lett. B 529 (2002) 187 [hep-ph/0102200] [INSPIRE].

[26] G. Duda, G. Gelmini, P. Gondolo, J. Edsjo and J. Silk, Indirect detection of a subdominant density component of cold dark matter, Phys. Rev. D 67 (2003) 023505 [hep-ph/0209266] [INSPIRE].

[27] S. Profumo, K. Sigurdson and L. Ubaldi, Can we discover multi-component WIMP dark matter?, JCAP 12 (2009) 016 [arXiv:0907.4374] [INSPIRE].

[28] X. Gao, Z. Kang and T. Li, The Supersymmetric Standard Models with Decay and Stable Dark Matters, Eur. Phys. J. C 69 (2010) 467 [arXiv:1001.3278] [InSPIRE].

[29] D. Feldman, Z. Liu, P. Nath and G. Peim, Multicomponent Dark Matter in Supersymmetric Hidden Sector Extensions, Phys. Rev. D 81 (2010) 095017 [arXiv:1004.0649] [INSPIRE].

[30] H. Baer, A. Lessa, S. Rajagopalan and W. Sreethawong, Mixed axion/neutralino cold dark matter in supersymmetric models, JCAP 06 (2011) 031 [arXiv:1103.5413] [INSPIRE].

[31] M. Aoki, M. Duerr, J. Kubo and H. Takano, Multi-Component Dark Matter Systems and Their Observation Prospects, Phys. Rev. D 86 (2012) 076015 [arXiv:1207.3318] [INSPIRE].

[32] D. Chialva, P.S.B. Dev and A. Mazumdar, Multiple dark matter scenarios from ubiquitous stringy throats, Phys. Rev. D 87 (2013) 063522 [arXiv:1211.0250] [INSPIRE].

[33] S. Bhattacharya, A. Drozd, B. Grzadkowski and J. Wudka, Two-Component Dark Matter, JHEP 10 (2013) 158 [arXiv:1309.2986] [INSPIRE].

[34] Y. Kajiyama, H. Okada and T. Toma, Multicomponent dark matter particles in a two-loop neutrino model, Phys. Rev. D 88 (2013) 015029 [arXiv:1303.7356] [INSPIRE].

[35] S. Esch, M. Klasen and C.E. Yaguna, A minimal model for two-component dark matter, JHEP 09 (2014) 108 [arXiv:1406.0617] [INSPIRE]. 
[36] K.R. Dienes and B. Thomas, Dynamical Dark Matter: I. Theoretical Overview, Phys. Rev. D 85 (2012) 083523 [arXiv: 1106.4546] [INSPIRE].

[37] K.R. Dienes and B. Thomas, Dynamical Dark Matter: II. An Explicit Model, Phys. Rev. D 85 (2012) 083524 [arXiv: 1107.0721] [InSPIRE].

[38] K.R. Dienes, S. Su and B. Thomas, Distinguishing Dynamical Dark Matter at the LHC, Phys. Rev. D 86 (2012) 054008 [arXiv: 1204.4183] [INSPIRE].

[39] K.R. Dienes, J. Kumar and B. Thomas, Direct Detection of Dynamical Dark Matter, Phys. Rev. D 86 (2012) 055016 [arXiv: 1208.0336] [INSPIRE].

[40] K.R. Dienes, J. Kumar and B. Thomas, Dynamical Dark Matter and the positron excess in light of AMS results, Phys. Rev. D 88 (2013) 103509 [arXiv:1306.2959] [InSPIRE].

[41] K.R. Dienes, S. Su and B. Thomas, Strategies for probing nonminimal dark sectors at colliders: The interplay between cuts and kinematic distributions, Phys. Rev. D 91 (2015) 054002 [arXiv: 1407.2606] [INSPIRE].

[42] G. Bélanger and J.-C. Park, Assisted freeze-out, JCAP 03 (2012) 038 [arXiv:1112.4491] [INSPIRE].

[43] O. Lebedev, H.M. Lee and Y. Mambrini, Vector Higgs-portal dark matter and the invisible Higgs, Phys. Lett. B 707 (2012) 570 [arXiv:1111.4482] [INSPIRE].

[44] K. Griest and M. Kamionkowski, Unitarity Limits on the Mass and Radius of Dark Matter Particles, Phys. Rev. Lett. 64 (1990) 615 [InSPIRE].

[45] H. Davoudiasl and I.M. Lewis, Dark Matter from Hidden Forces, Phys. Rev. D 89 (2014) 055026 [arXiv: 1309.6640] [INSPIRE].

[46] Y. Hochberg, E. Kuflik, H. Murayama, T. Volansky and J.G. Wacker, The SIMPlest Miracle, arXiv: 1411.3727 [INSPIRE].

[47] Y. Hochberg, E. Kuflik, T. Volansky and J.G. Wacker, Mechanism for Thermal Relic Dark Matter of Strongly Interacting Massive Particles, Phys. Rev. Lett. 113 (2014) 171301 [arXiv: 1402.5143] [INSPIRE].

[48] N. Yamanaka, S. Fujibayashi, S. Gongyo and H. Iida, Dark matter in the hidden gauge theory, arXiv: 1411.2172 [INSPIRE].

[49] X.W. Liu, X.F. Wu and T. Lu, GRB 060206: hints of precession of the central engine?, Astron. Astrophys. 487 (2008) 503 [arXiv:0808.1172] [INSPIRE].

[50] P.H. Frampton and B.-H. Lee, SU(15) grand unification, Phys. Rev. Lett. 64 (1990) 619 [INSPIRE].

[51] T. Hambye, Hidden vector dark matter, JHEP 01 (2009) 028 [arXiv:0811.0172] [INSPIRE].

[52] T.W.B. Kibble, Some Implications of a Cosmological Phase Transition, Phys. Rept. 67 (1980) 183 [INSPIRE].

[53] T.W.B. Kibble, Topology of Cosmic Domains and Strings, J. Phys. A 9 (1976) 1387 [INSPIRE].

[54] Y. Zeldovich, I.Y. Kobzarev and L.B. Okun, Cosmological Consequences of the Spontaneous Breakdown of Discrete Symmetry, Zh. Eksp. Teor. Fiz. 67 (1974) 3 [INSPIRE].

[55] M. Gonderinger, H. Lim and M.J. Ramsey-Musolf, Complex Scalar Singlet Dark Matter: 
Vacuum Stability and Phenomenology, Phys. Rev. D 86 (2012) 043511 [arXiv:1202.1316] [INSPIRE].

[56] V. Barger, P. Langacker, M. McCaskey, M. Ramsey-Musolf and G. Shaughnessy, Complex Singlet Extension of the Standard Model, Phys. Rev. D 79 (2009) 015018 [arXiv:0811.0393] [INSPIRE].

[57] A. Biswas, D. Majumdar, A. Sil and P. Bhattacharjee, Two Component Dark Matter: A Possible Explanation of $130 \mathrm{GeV} \gamma-$ Ray Line from the Galactic Centre, JCAP 12 (2013) 049 [arXiv: 1301.3668] [INSPIRE].

[58] J. Edsjo and P. Gondolo, Neutralino relic density including coannihilations, Phys. Rev. D 56 (1997) 1879 [hep-ph/9704361] [INSPIRE].

[59] Particle Data Group collaboration, K.A. Olive et al., Review of Particle Physics, Chin. Phys. C 38 (2014) 090001.

[60] R.D. Young and A.W. Thomas, Octet baryon masses and sigma terms from an $\mathrm{SU}(3)$ chiral extrapolation, Phys. Rev. D 81 (2010) 014503 [arXiv:0901.3310] [INSPIRE].

[61] LUX collaboration, D.S. Akerib et al., First results from the LUX dark matter experiment at the Sanford Underground Research Facility, Phys. Rev. Lett. 112 (2014) 091303 [arXiv: 1310.8214] [INSPIRE].

[62] LeP Working Group for Higgs boson searches, ALEPH, DELPHI, L3, OPAL collaboration, R. Barate et al., Search for the standard model Higgs boson at LEP, Phys. Lett. B 565 (2003) 61 [hep-ex/0306033] [INSPIRE].

[63] Z. Kang, J. Li, T. Li, D. Liu and J. Shu, Probing the CP-even Higgs sector via $\mathrm{H}_{3} \mathrm{H}_{2} \mathrm{H}_{1}$ in the natural next-to-minimal supersymmetric standard model, Phys. Rev. D 88 (2013) 015006 [arXiv: 1301.0453] [INSPIRE].

[64] R.S. Chivukula, A. Farzinnia, J. Ren and E.H. Simmons, Constraints on the Scalar Sector of the Renormalizable Coloron Model, Phys. Rev. D 88 (2013) 075020 [arXiv:1307.1064] [INSPIRE].

[65] R. Khosravi and F. Falahati, Semileptonic decays of $B_{s}$ to $\phi$ meson in QCD, Phys. Rev. D 88 (2013) 056002 [INSPIRE].

[66] G. Degrassi et al., Higgs mass and vacuum stability in the Standard Model at NNLO, JHEP 08 (2012) 098 [arXiv: 1205.6497] [INSPIRE].

[67] J. Fleischer and F. Jegerlehner, Radiative Corrections to Higgs Decays in the Extended Weinberg-Salam Model, Phys. Rev. D 23 (1981) 2001 [INSPIRE].

[68] A. Sirlin and R. Zucchini, Dependence of the Quartic Coupling $H(m)$ on $M(H)$ and the Possible Onset of New Physics in the Higgs Sector of the Standard Model, Nucl. Phys. B 266 (1986) 389 [INSPIRE].

[69] M.J.G. Veltman, The Infrared - Ultraviolet Connection, Acta Phys. Polon. B 12 (1981) 437 [INSPIRE].

[70] P. Gondolo and G. Gelmini, Cosmic abundances of stable particles: Improved analysis, Nucl. Phys. B 360 (1991) 145 [inSPIRE].

[71] K.P. Modak, D. Majumdar and S. Rakshit, A Possible Explanation of Low Energy $\gamma$-ray Excess from Galactic Centre and Fermi Bubble by a Dark Matter Model with Two Real Scalars, JCAP 03 (2015) 011 [arXiv:1312.7488] [INSPIRE]. 